\title{
A Three-Dimensional Nonlinear Timoshenko Beam Based on the Core-Congruential Formulation
}

\author{
Luis A. CRIVELLI \\ Carlos A. Felippa \\ Department of Aerospace Engineering Sciences and \\ Center for Space Structures and Controls \\ University of Colorado \\ Boulder, Colorado 80309-0429, USA
}

May 1992

Report No. CU-CSSC-92-05

\begin{abstract}
E.
Submitted to International Journal Numerical Methods in Engineering

Research supported by Air Force Office of Scientific Research (AFOSR) under Grant F49620-87-C-0074, the National Science Fundation under Grant 8717773, NASA Langley Research Center under Grant NAS1-756, and NASA UERC Program under the Center for Space Construction.
\end{abstract}




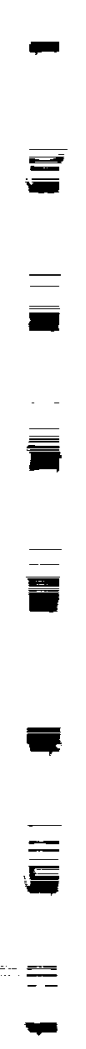

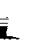
:

-

$$
\text { ? }
$$




\title{
A Three-Dimensional Nonlinear Timoshenko Beam Based on the Core-Congruential Formulation
}

\author{
L. A. Crivelli and C. A. Felippa \\ Department of Aerospace Engineering and \\ Center for Space Structures and Controls \\ University of Colorado at Boulder \\ Boulder, Colorado 80909-0429, USA
}

\begin{abstract}
SUMMARY
A three-dimensional, geometrically nonlinear two-node Timoshenko beam element based on the Total Lagrangian description is derived. The element behavior is assumed to be linear elastic, but no restrictions are placed on magnitude of finite rotations. The resulting element has twelve degrees of freedom: six translational components and six rotational-vector components. The formulation uses the Green-Lagrange strains and second Piola-Kirchhoff stresses as energy-conjugate variables and accounts for for bending-stretching and bending-torsional coupling effects without special provisions. The core-congruential formulation (CCF) is used to derived the discrete equations in a staged manner. Core equations involving the internal force vector and tangent stiffness matrix are developed at the particle level. A sequence of matrix transformations carries these equations to beam cross-sections and finally to the element nodal degrees of freedom. The choice of finite rotation measure is made in the next-to-last transformation stage, and the choice of over-the-element interpolation in the last one. The tangent stiffness matrix is found to retain symmetry if the rotational vector is chosen to measure finite rotations. An extensive set of numerical examples are presented to test and validate the present element.
\end{abstract}



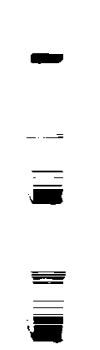

$\bar{E}$

豆

$\bar{E}$

$\bar{E}$

$=$

$=$

$-$

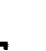

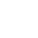

$-$

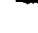

$=$

$=$

-

$\underline{\overline{\underline{E}}}$ 


\section{INTRODUCTION}

The computer-based geometrically-nonlinear analysis of flexible three-dimensional structures has attracted considerable interest in recent years. In the aerospace field, part of this attention comes from establishing challenging and ambitious goals for space research, such as the space station, space-based antennas for improved communications, space-based telescopes, solar arrays, and a large variety of similar devices which have been given detailed consideration in various proposals for future space developments. In mechanical engineering, interest has emerged from the competition to obtain reliable, accurate and inexpensive manufacturing procedures, especially in the development of the new generation of robots capable of performing high precision tasks such as microspot welding and assembly of orbiting structures. Further interest comes from analysis and design of high-performance aircraft, helicopter blades and turbomachinery. These applications have motivated the development of more physically realistic computational models of large flexible structures that exhibit pronounced geometric nonlinearities.

Three kinematic descriptions have been used in geometrically nonlinear finite element analysis: Total Lagrangian, Updated Lagrangian and corotational. The present work follows the Total Lagrangian (TL) description, but in an unconventional variant that constructs the nonlinear finite element equations in a staged fashion. This variant is called the corecongruential formulation and identified by the acronym CCF in the sequel. An account of this methodology is presented in a recent review paper by Felippa and Crivelli. ${ }^{1}$ This review concludes that a key advantage of the CCF for constructing TL elements is that it helps establishing consistency by avoiding the premature introduction of drastic kinematic approximations.

The main ideas behind the CCF can be traced to a 1973 paper by Rajasekaran and Murray $^{2}$, who examined critically the pioneer work on the Total Lagrangian description by Mallett and Marcal ${ }^{3}$. The 1974 discussion of Rajasekaran-Murray's paper by Felippa ${ }^{4}$ established general expressions for the finite element equations that appeared at various variational levels. Further historical details are given in the review by Felippa and Crivelli. ${ }^{1}$

This paper uses the CCF to derive the finite element equations of a TL three-dimensional Timoshenko beam element that can undergo arbitrarily large rotations. First, we derive the governing differential equations encountered in the geometrically nonlinear static structural analysis of three-dimensional beams. Next, the finite element counterparts are obtained by discretizing the physical degrees of freedom. Our main assumption is that the beam behaves 
as a linear hyperelastic isotropic medium, which allows us to write its internal energy as a quadratic function of the finite strains. We obtain the equilibrium equations from the stationarity condition on the first variation of the total energy. Similarly, we obtain the rate or incremental equations from the second variation of the total energy.

The CCF derivation of the governing equations of motion proceeds through two phases: a core phase followed by a transformation phase. In the initial phase core energy, residual and tangent stiffness matrices as well as internal force vectors, are obtained independently of any specific choice used to represent or parametrize the motion. These matrices and vectors pertain to individual particles. They do not depend on discretization decisions, such as element geometry, shape functions and selection of nodal degrees of freedom. To emphasize this independence, the term core was coined. In the transformation phase, these core forms are gradually specialized to particular element instances. This specialization is achieved by the application of one or more transformation stages that progressively "bind" particles into lines, areas or volumes through kinematic constraints, and eventually link the element domain to the nodal degrees of freedom. The choice of specific parametrizations for finite rotations may be deferred to latter stages.

What are the differences between the CCF and the more conventional Total Lagrangian formulation of nonlinear finite elements? If kinematic exactness is maintained throughout, the final discrete equations are identical. But in geometrically nonlinear analysis approximations of various kinds are common, especially in structural elements with rotational degrees of freedom such as beams, plates and shells. In the conventional, one-shot formulation it is often difficult to assess a priori the effect of seemingly innocuous approximations "thrown into the pot," and a posteriori exhaustive testing of complex situations becomes virtually impossible. Sample: how does the neglect of higher order terms in the axial deformation of a spinning beam affects torsional buckling?

The staged approach taken in the CCF permits a better control over such assumptions. The core equations are physically transparent, clearly displaying the effect of material behavior, displacement gradients and prestresses. In the ensuing transformation sequence the origin of each term can be exactly traced, and on that basis informed decisions on retention or dropping made.

Another important advantage of the staged approach is the precise identification (and avoidance) of kinematic choices that lead to unsymmetries in the tangent stiffness. In beams, plates and shell elements such a symmetry loss is linked to the choice of the finite-rotation 
parametrization (Euler angles, Euler parameters, rotational vector, direction cosines, etc). This decision can in fact be postponed to the final stages, and changed if necessary without affecting "kernel" forms derived in previous stages. This nesting has obvious beneficial influence on element programming modularity.

\section{PREVIOUS WORK IN NONLINEAR SPACE STRUCTURES}

Early work in the area of orbiting and free-flying structures dealt with the problem of rigid spacecrafts with flexible appendages attached to their core. ${ }^{5}$ In this case, the motion of the system was obtained by superposing a given number of linear elastic modes to the overall motion obtained considering the structure as made of interconnected rigid bodies. This procedure has been called the hybrid coordinate method. ${ }^{6}$ The flexible motion -assumed small - is then described with respect to frames attached to the underlying rigid core motion.

When this procedure is to be applied to structures with distributed flexibility - those not having a distinct rigid core - a question that immediately arises is how to choose the reference frame. One idea is to define a floating or unattached frame that is optimum in some sense. Two frames were proposed by De Veubeke, ${ }^{7}$ one that minimizes the relative kinetic energy and another that minimizes the deformation energy. The first choice is shown to correspond to Tisserand's conditions of zero relative momentum and angular momentum, i.e., the rigid body modes are found to be fixed in this frame. However, this choice introduces some practical difficulties, especially in the case where there are lumped sources of kinetic energy, such as rotating masses or gyros. Further work has also been done to include the effects of spinning rotors. ${ }^{8}$ The hybrid coordinate method has the advantage that the equations of motion are represented in a form similar to rigid body equations. ${ }^{9}$ This type of moving frame has also been called mean axis system ${ }^{10}$ and used to implement finite elements representations. Extensions of the mean axis formulation to flexible multibody system dynamics can also be found. ${ }^{11}$ De Veubeke $^{7}$ shows that the use of the minimum deformation energy criterion allows the relative displacement to be exactly represented by an expansion in natural elastic vibration modes and leads to a simpler implementation. In any case, introducing a floating frame requires constraint equations to be added, because they require the definition of additional variables that cannot be obtained directly from the dynamics of the system.

A more subtle problem associated with the floating frame formulation is found in connection with spinning free-free beams. ${ }^{12}$ In this case, nonlinear effects produce a geometric stiffening due to the spin-induced longitudinal stretch. The resulting axial force, which cannot be considered infinitesimal, affects the beam bending stiffness, showing that the uncoupling 
between rigid body modes and elastic modes no longer holds. Several attempts have been made to include these and other effects by higher order corrections to the theory. ${ }^{13}$ This leads to cumbersome expressions for the matrices entering the governing equations of motion, without apparent advantages with respect to a full nonlinear theory.

With increasing interest in control-structure interaction, the floating frame approach has gained new attention, especially when the flexible component is attached to a large rigid mass and there is a hierarchical control system that keeps the elastic deformation small. A typical example is that of a flexible beam or antenna attached to the space shuttle. ${ }^{14}$ In this case, the shuttle can be regarded as a rigid body to which the reference frame is attached, while the flexible part is discretized using finite elements. The relative equations of motion of the flexible part are linear whereas the nonlinearity comes from the coupling with the rigid body motion. Assuming that the inertia of the flexible part is small compared to the inertia of the shuttle, the flexible motion can be regarded as a perturbation to the rigid body motion. This perturbation technique allows the analyst to define a rigid-body maneuvering strategy independently of the elastic behavior. The linearity of the elastic component is required to construct an optimal feedback control scheme for vibration arrest. This methodology has been used to model the SCOLE experiment. ${ }^{15}$

When performing large-rotation dynamics analysis using the floating frame approach, it is important to note that the coupling with the rigid body motion must come through the inertia components, because the deformation components have been intentionally uncoupled from it. Thus, the inherent nonlinearity of the problem carries over to the inertia terms, leading to fully populated nonlinear mass matrices that ruin the sparsity property of conventional finite element analysis. For large systems, this is computationally inefficient and prohibitive in terms of storage, forcing analysts who use this approach to look for reduction methods. Thus, most of the programs based on this approach use linear modes of the free-free structure in the undeformed configuration to condense the problem to a few degrees of freedom. Another approach is to use a fixed frame for the inertial terms only. ${ }^{16,17}$

Although the single frame approach has been used extensively in spacecraft, its applications are limited to small elastic deformations and thus mainly confined to the modeling of free-vibration dynamics. When the primary concern is large deflections, as in the case of stability and/or postbuckling analyses, the relative rotations between structural components are no longer infinitesimal. It is also desirable to preserve the structure of the finite element equations for problems of dynamic instability. This has motivated the development of the 
corotational description. This description retains multiple reference frames relative to which the elastic deformation of portions of the structure are described. ${ }^{18}$ In its combination with finite elements each one has a co-moving attached Cartesian frame. The motion of this rigid frame is used to decompose the element displacements into rigid body and deformational components. Because the latter are assumed small in each element, small relative deformation measures may be used. This model is intimately related to the discretization process, i.e., the finite element discretization is done before the establishment of the equations of motion or the definition of the variational principle. This procedure has been extended to dynamics analysis of space frames ${ }^{19}$ but only limited to explicit integration schemes, which do not require the explicit computation of the stiffness matrix.

Because of the small relative deformation assumptions, the expressions of the finite element matrices in the corotational frame can be those corresponding to a linear finite element model, optionally corrected by geometric stiffness effects. An interesting question that may be raised is: Is it possible to obtain a set of external transformations that project these matrices into the global frame? This will have the advantage that existing finite elements can be taken as a "core" component which can be transformed to the global equations by appropriate external manipulations. The answer given by Rankin and coworkers is partially positive. ${ }^{20,21}$ This was done by enforcing rotational invariance of the internal force, which in turns translates into the satisfaction of rotational equilibrium. This technique relies on the use of a projector operator which removes the rigid rotations. However, the kinematics properties of the corotated frame still depend on a subset of element properties such as dimensionality and the number of nodal points.

Another way to achieve the projection goal is to use a finite strain theory from the outset. In this case, the effect of large rotations is automatically taken into account. Simo and coworkers ${ }^{22,23}$ and Cardona ${ }^{24}$ have exploited the first Piola-Kirchhoff (PK1) stress, for which the conjugate strain is simply the deformation gradient. This leads to a relatively straightforward formulation of the discrete equilibrium equations, from which an incremental solution procedure is obtained. Downer Park and Chiou ${ }^{25}$ have constructed a corotational formulation based on Cauchy (true) stress increments and applied to the dynamic analysis of spinning beams, with emphasis on energy and momentum conserving algorithms.

The present work differs from previous ones in the following respects:

1. The Total Lagrangian (TL) description is used for 3D Timoshenko beam elements in conjunction with the second Piola-Kirchhoff (PK2) stress and the Green-Lagrange (GL) 
strain. A symmetric tangent stiffness is obtained for a particular choice of the finiterotation measure.

2. No kinematic restrictions are placed on the overall rotations. Only a mild restriction applies at the element level: the relative rotations within an individual beam element should not exceed $360^{\circ}$.

3. The CCF is used in the element derivation.

Sections 3 introduces basic terminology while Sections 4-6 describe the CCF in general terms. The formulation is then applied to the three-dimensional beam element in Sections 7-11. Section 12 present numerical examples.

\section{NONLINEAR MATRIX EQUATIONS}

In this section we summarize the discrete governing equations of a geometrically nonlinear structure expressed in terms of a set of generalized coordinates $q$ that for the moment are left unspecified. The resulting quadratic forms in $\mathbf{q}$ contain deformation-dependent kernel matrices collectively called stiffness matrices. This deformation dependency changes with the variational level. In the sequel we examine variational levels 0,1 and 2 , otherwise identified as the energy, residual-force equilibrium, and incremental levels, respectively.

Variational Level 0: Potential Energy. The internal energy $U$ is a nonlinear function of the generalized coordinates formally expressable as

$$
U=\frac{1}{2} \mathbf{q}^{T} \mathbf{K}^{U} \mathbf{q}+\mathbf{q}^{T} \mathbf{p}^{0}
$$

The component of $U$ that is linear in $\mathbf{q}$ is the prestress force vector $\mathbf{p}^{0}$. The component of $U$ that is quadratic and higher in the freedoms $\mathbf{q}$ is assigned the kernel $\mathbf{K}^{U}$. This is a symmetric matrix with dimensions of stiffness, called the energy stiffness. The total potential energy is

$$
J=U-\mathbf{q}^{T} \mathbf{p}
$$

where $\mathbf{p}$ is the vector of applied generalized forces conjugate to $\mathbf{q}$. Throughout the present work the applied loads $\mathbf{p}$ are assumed to be conservative and deformation independent.

Variational Level 1: Residual Force Equilibrium. The first variation $\delta U=\mathbf{f}^{T} \delta \mathrm{q}$ of the strain energy defines the internal force vector $\mathbf{f}=\partial U / \partial \mathbf{q}$. Under certain conditions studied later this vector may be expressed as

$$
\mathbf{f}=\frac{\partial U}{\partial \mathbf{q}}=\mathbf{K}^{r} \mathbf{q}+\mathbf{p}^{0}
$$


This relation defines the secant stiffness matrix $\mathbf{K}^{r}$, which (if it exists) is generally unsymmetric. The force residual is the difference between internal and external forces:

$$
\mathbf{r}=\mathbf{f}-\mathbf{p}
$$

Setting $\mathbf{r}$ to zero gives the discrete equilibrium equations.

Variational Level 2: Incremental Equilibrium. The force equilibrium equation $\mathbf{r}=0$ is nonlinear in $\mathbf{q}$. This equation is usually treated numerically by continuation procedures that search for solutions in the neighborhood of a previously computed equilibrium point. To implement this technique the residual is expressed as a function of $q$ and a continuation parameter $\lambda$ that parametrizes the applied forces:

$$
\mathbf{r}(\mathbf{q}, \lambda)=\mathbf{f}(\mathbf{q})-\mathbf{p}(\lambda)=\mathbf{0}
$$

Differentiating with respect to $\lambda$ yields the first-order rate equations

$$
\mathbf{r}^{\prime}=\mathbf{K} \mathbf{q}^{\prime}-\mathbf{p}^{\prime}=\mathbf{0},
$$

where primes denote differentiation with respect to $\lambda$. Multiplying by $d \lambda$ and converting the $d$ 's to $\Delta$ 's gives the popular incremental form $K \Delta \mathbf{q}=\Delta \mathbf{p}$.

The tangent stiffness is fundamental in incremental-iterative solution methods and stability analysis, whereas the secant stiffness (by itself or embedded in the internal force vector $f$ ) is important in pseudo-force methods. The energy stiffness enjoys limited application per se but has theoretical importance as source for the other two. In the sequel we use the notation $\mathbf{K}^{\text {level }}$ to collectively designate these three matrices.

\section{CORE PHASE OF CCF}

The core phase of the CCF establishes nonlinear response equations at the particle level, using the displacement gradients as degrees of freedom. The resulting equations depend on the mathematical model under consideration - bar, beam, plate, shell, 3D continuum, etc. - insofar as the form of the internal energy density, but are otherwise independent of finite element discretization decisions.

Under the effect of conservative loads the structure displaces from a reference configuration $\mathcal{C}_{0}$, with particle coordinates $X_{i}$, to a variable current configuration $\mathcal{C}$, with corresponding particle coordinates $x_{i}$. The particle displacements $u_{i}=x_{i}-X_{i}$ are collected in $\mathbf{u}$. Let the 
state of strain at $\mathcal{C}$ be characterize by $n_{s}$ strain components $e_{i}$ collected in array e, and let the $n_{s}$ conjugate stresses be $s_{i}$, collected in array s.

We assume that strains stay small so that the structure remains linearly elastic. Using the summation convention the elastic stress-strain relations may be written

$$
s_{i}=s_{i}^{0}+E_{i j} e_{i}, \quad \text { or } \mathbf{s}=\mathbf{s}^{0}+E \mathbf{e}
$$

where $s_{i}^{0}$ are stresses in $\mathcal{C}_{0}$ (stresses that remain if $e_{i}=0$, also called prestresses) and $E_{i j}=E_{j i}$ are elastic moduli arranged as a symmetric matrix $\mathbf{E}$ in the usual manner. The strain energy density may be written

$$
\mathcal{U}=e_{i} s_{i}^{0}+\frac{1}{2} e_{i} E_{i j} e_{j}=\mathbf{e}^{T} \mathbf{s}^{0}+\frac{1}{2} \mathbf{e}^{T} \text { Ee. }
$$

The total strain energy $U$ is obtained by integrating (8) over the structure volume: $U=$ $\int_{V_{0}} \mathcal{U} d V_{0}$; the integration taking place - as can be expected in a TL description - over the reference configuration geometry.

Introduce now the $n_{g}$ displacement gradients $g_{m n}=\partial u_{m} / \partial X_{n}$. These are alternatively identified as $g_{i}\left(i=1,2, \ldots n_{g}\right)$ so they can be conveniently arranged in a one-dimensional array g. Following Rajasekaran and Murray ${ }^{2}$ and Felippa ${ }^{4}$, assume that the strains $e_{i}$ are linked to the displacement gradients through matrix relations of the form

$$
e_{i}=\mathbf{h}_{i}^{T} \mathbf{g}+\frac{1}{2} \mathbf{g}^{T} \mathbf{H}_{i} \mathbf{g}, \quad i=1,2, \ldots n_{s}
$$

where $\mathbf{h}_{i}$ and $\mathbf{H}_{\boldsymbol{i}}$ are arrays of dimension $n_{g} \times 1$ and $n_{g} \times n_{g}$, respectively, with $\mathbf{H}_{i}$ symmetric. If the Green-Lagrange (GL) strain measure is chosen, all $\mathbf{H}_{i}$ are independent of $g$, a restriction enforced throughout this work.

Denote by $\mathbf{S}^{U}, \mathbf{S}^{r}$ and $\mathbf{S}$ the energy, secant and principal tangent core stiffness matrices, respectively, where the qualifier "principal" is explained in Sections 5-6. Symbols $\Phi$ and $\Psi^{0}$ denote the core counterpart of the force vectors $\mathbf{f}$ and $\mathbf{p}^{0}$. With this notation the first and second variations of the strain energy density can be expressed as

$$
\begin{aligned}
& \delta \mathcal{U}=\delta \mathbf{g}^{T}\left(\mathbf{S}^{U} \mathbf{g}+\boldsymbol{\Psi}^{0}\right)+\frac{1}{2} \mathbf{g}^{T} \delta \mathbf{S}^{U} \mathbf{g}=\delta \mathbf{g}^{T}\left(\mathbf{S}^{r} \mathbf{g}+\Psi^{0}\right)=\delta \mathbf{g}^{T} \boldsymbol{\Phi} \\
& \delta^{2} \mathcal{U}=\delta \mathbf{g}^{T} \mathbf{S}^{r} \delta \mathbf{g}+\delta \mathbf{g}^{T} \delta \mathbf{S}^{r} \mathbf{g}+\left(\delta^{2} \mathbf{g}\right)^{T} \boldsymbol{\Phi}=\delta \mathbf{g}^{T} \mathbf{S} \delta \mathbf{g}+\left(\delta^{2} \mathbf{g}\right)^{T} \boldsymbol{\Phi}
\end{aligned}
$$

These variational equations implicitly determine $\mathbf{S}^{r}, \boldsymbol{\Phi}$ and $\mathbf{S}$ from $\mathbf{S}^{U}$ and $\Psi^{0}$. 


\section{SPECTRAL FORMS}

General expressions for $\mathbf{S}^{U}, \mathbf{S}^{r}, \mathbf{S}$ and $\mathbf{\Phi}$ are given in Felippa and Crivelli ${ }^{1}$ where it is noted that both $\mathbf{S}^{U}$ and $\mathbf{S}^{r}$ may have arbitrary coefficients. More compact expressions called spectral forms (because of their formal similarity with the spectral decomposition of a matrix as the sum of rank-one matrices) can be presented at the cost of some generality. Introduce the vectors

$$
\mathbf{c}_{i}=\mathbf{h}_{i}+\frac{1}{2} \mathbf{H}_{i} \mathbf{g}, \quad \mathbf{b}_{i}=\frac{\partial e_{i}}{\partial \mathbf{g}}=\mathbf{h}_{i}+\mathbf{H}_{i} \mathbf{g} .
$$

Then $e_{i}=\mathbf{c}_{i}^{T} \mathbf{g}, s_{i} e_{i}=E_{i j} \mathbf{g}^{T} \mathbf{c}_{i}^{T} \mathbf{c}_{j} \mathbf{g}$, and the spectral forms, with the summation convention implied throughout, are

$$
\begin{gathered}
\mathbf{S}^{U}=E_{i j} \mathbf{c}_{i} \mathbf{c}_{j}+s_{i}^{0} \mathbf{H}_{i}, \\
\mathbf{S}^{r}=E_{i j} \mathbf{c}_{i} \mathbf{c}_{j}+\frac{1}{2}\left(s_{i}^{0}+s_{i}\right) \mathbf{H}_{i}, \\
\Phi=s_{i} \mathbf{b}_{i}, \\
\mathbf{S}=\frac{\partial \Phi}{\partial \mathbf{g}}=E_{i j} \mathbf{b}_{i} \mathbf{b}_{j}+s_{i} \mathbf{H}_{i}=\mathbf{S}_{M}+\mathbf{S}_{G P} .
\end{gathered}
$$

The decomposition (16) of the principal tangent core stiffness $\mathbf{S}$ should be noted:

1. The material stiffness matrix $\mathbf{S}_{M}=\left(\partial s_{i} / \partial \mathbf{g}\right) \mathbf{b}_{i}=E_{i j} \mathbf{b}_{i} \mathbf{b}_{j}^{T}$, which depends on the constitutive coefficients and displacement gradients.

2. The principal geometric stiffness matrix $\mathrm{S}_{G P}=s_{i}\left(\partial \mathrm{b}_{i} / \partial \mathrm{g}\right)=s_{i} \mathrm{H}_{i}$, which depends on the current PK2 stresses.

The second variation (11) of the internal energy density has $\mathbf{S}=\mathbf{S}_{M}+\mathbf{S}_{G P}$ as kernel of the quadratic form in $\delta$ g. The core internal force $\Phi$ also appears in the inner product $\left(\delta^{2} \mathbf{g}\right)^{T} \boldsymbol{\Phi}$. This second term may either survive or drop out depending on the relation of $\mathbf{g}$ with the target physical or generalized coordinates chosen in the CCF transformation phase, as discussed in the following section.

If the term drops out, $\mathbf{S}$ becomes the tangent core stiffness and the qualifier "principal" becomes superfluous. If the term survives, it contributes to what we call the complementary geometric stiffness.

\section{TRANSFORMATION PHASE OF CCF}

The core stiffness matrices and force vectors given in (13)-(16) pertain to each material particle of the structure. They can be used to construct physical stiffness matrices and 
force vectors through a process involving core-to-physical transformation and integration. This transformation phase of the CCF converts the core equations to discrete equations in terms of physical or generalized degrees of freedom collected in vector $\mathbf{q}$, which defines the mechanical behavior of a reference volume $V_{0}$ (which may specialize to a line or area domain).

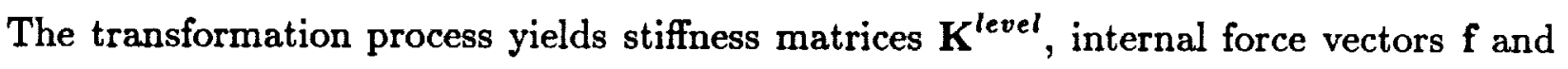
prestress forces $\mathbf{p}^{0}$. The effect of these transformations depend on the nature of the relations between the source core variables $\mathbf{g}$, and the target variables $\mathbf{q}$, which for the moment are assumed to be independent. Three possibilities exist.

Case I. Linear relation between $\mathbf{g}$ and $\mathbf{q}$. We have $\mathbf{g}=\mathbf{T q}$, or in indicial form $g_{i}=T_{i j} q_{j}$, where $\mathbf{T}$ is a transformation matrix independent of $\mathbf{q}$. Invariance of $U$ and its two variations taken over the reference volume $V_{0}$ yields

$$
\mathbf{K}^{\text {level }}=\int_{V_{0}} \mathbf{T}^{T} \mathbf{S}^{\text {level }} \mathbf{T} d V_{0}, \quad \mathbf{f}=\int_{V_{0}} \mathbf{T}^{T} \mathbf{\Phi} d V_{0}, \quad \mathbf{p}^{0}=\int_{V_{0}} \mathbf{T}^{T} \Psi^{0} d V_{0} .
$$

The stiffness transformations at all levels are congruential, which gives the standard CCF its name. This is the case for continuum and structural finite elements that possess only translational degrees of freedom. Two examples of such elements are given in the survey by Felippa and Crivelli ${ }^{1}$.

Case II. Nonlinear algebraic relation between $\mathbf{g}$ and $\mathbf{q}$. We have $\mathbf{g}=\mathbf{g}(\mathbf{q})$ or in index notation, $g_{i}=g_{i}\left(q_{j}\right)$. Differentiating with respect to the $q_{i}$ variables yields

$$
\begin{aligned}
\delta g_{i} & =\frac{\partial g_{i}}{\partial q_{j}} \delta q_{j}=T_{i j} \delta q_{j}, \quad \text { or } \delta \mathbf{g}=\mathbf{T} \delta \mathbf{q} \\
\delta^{2} g_{i} & =\frac{\partial^{2} g_{i}}{\partial q_{j} \partial q_{k}} \delta q_{j} \delta q_{k}+\frac{\partial g_{i}}{\partial q_{j}} \delta^{2} و_{\zeta} \nearrow^{0}=F_{i j k} \delta q_{j} \delta q_{k}, \quad \text { or } \delta^{2} \mathbf{g}=(\mathbf{F} \delta \mathbf{q}) \delta \mathbf{q}
\end{aligned}
$$

where $(\mathbf{F} \delta \mathbf{q})$ is the matrix $F_{i j k} \delta q_{k}=F_{i j k} \delta q_{j} ; \mathbf{F}$ being a cubic array. The second term in the expansion of $\delta^{2} q_{i}$ vanishes because the $q_{i}$ are assumed to be independent variables. Invariance of $\delta^{2} U$ yields the tangent stiffness transformation

$$
\mathbf{K}=\int_{V_{0}}\left\{\mathbf{T}^{T}\left(\mathbf{S}_{M}+\mathbf{S}_{G P}\right) \mathbf{T}+\mathbf{Q}\right\} d V_{0}=\mathbf{K}_{M}+\mathbf{K}_{G P}+\mathbf{K}_{G C}=\mathbf{K}_{M}+\mathbf{K}_{G}
$$

where the entries of $\mathbf{Q}$ are $Q_{j k}=Q_{k j}=F_{i j k} \Phi_{i}$; note that $\mathbf{Q}$ is symmetric because $F_{i j k}=F_{i k j}$. Integration of $\mathbf{Q}$ over $V_{0}$ yields the complementary portion $\mathbf{K}_{G C}$ of the geometric stiffness $K_{G}$. The force vectors $\mathbf{f}$ and $\mathbf{p}^{0}$ are given by the last two expressions in (17) with $\mathbf{T}$ defined in (18). 
What happens to $\mathbf{K}^{U}$ and $\mathbf{K}^{r}$ ? They can be obtained, somewhat artificially, by defining $\mathbf{g}=\mathbf{G q}$ where $G_{i j}=g_{i} / q_{j}$ with some care given to the limits $q_{j} \rightarrow 0$. Then $\mathbf{K}^{U}=$ $\int_{V_{0}} \mathbf{G}^{T} \mathbf{S}^{U} \mathbf{G} d V_{0}$ and $\mathbf{K}^{r}=\int_{V_{0}} \mathbf{T}^{T} \mathbf{S}^{r} \mathbf{G} d V_{0}$. Because in general $\mathbf{T} \neq \mathbf{G}$ one cannot expect symmetry in the secant stiffness.

This case occurs in finite elements with degrees of freedom that are fixed-axis rotations, because plane rotations are integrable. Examples are provided by two-dimensional beams, and plane stress elements with drilling freedoms with only in-plane motions considered.

Case III. Differential relation between $\delta \mathbf{g}$ and $\delta \mathbf{q}$. In this final case only the variations of $\mathbf{g}$ and $q$ can be connected:

$$
\begin{aligned}
\delta g_{i} & =T_{i j} \delta q_{j}, \quad \text { or } \delta \mathbf{g}=\mathbf{T} \delta \mathbf{q}, \\
\delta^{2} g_{i} & =\frac{\partial T_{i j}}{\partial q_{k}} \delta q_{j} \delta q_{k}=F_{i j k} \delta q_{j} \delta q_{k} \quad \text { or } \delta^{2} \mathbf{g}=(\mathbf{F} \delta \mathbf{q}) \delta \mathbf{q}
\end{aligned}
$$

Transformation equations (19) still applies for $\mathbf{K}$ and (17) for $\mathbf{f}$ and $\mathbf{p}^{0}$. But no integral $\mathbf{g}=\mathbf{g}(\mathbf{q})$ as in Case II exists. Consequently $\mathbf{K}^{U}$ and $\mathbf{K}^{r}$, which need a "secant" relation $\mathbf{g}=\mathbf{G q}$, cannot be constructed. Furthermore $\mathbf{Q}$ is not necessarily symmetric; a condition for that being $F_{i j k}=F_{i k j}$ or $\partial T_{i j} / \partial q_{k}=\partial T_{i k} / \partial q_{j}$. Case III occurs when three-dimensional finite rotations appear as degrees of freedom, as in the present development.

Up to this point the $q$ have been assumed to be independent variables. But for complicated elements, such as the present one, the CCF transformations are more conveniently applied in stages because of the reasons noted in the Introduction. The target variables in one stage become the source variables for the next one.

What happens if the $q$ are intermediate variables in a transformation chain? If $q$ are linear in the final independent degrees of freedom $\mathbf{v}$, all previous formulas hold because Case I applies to the remaining transformations, which are strictly congruential. But if the $\mathbf{q}$ are nonlinear in $\mathbf{v}$ or only a nonintegrable differential relation exists, term $\left(\partial g_{i} / \partial q_{j}\right) \delta^{2} q_{j}=T_{i j} \delta^{2} q_{j}$ in the second of (18) survives. The net effect is that the complementary geometric stiffness acquires a higher order component, implicitly defined as the kernel of

$$
\int_{V_{0}} \Phi_{i} T_{i j} \delta^{2} q_{j} d V_{0}
$$

This term cannot be resolved (meaning the explicit extraction of its stiffness kernel) until the transformation chain reaches downstream variables that either are the final degrees of freedom (and thus independent), or depend linearly on such. It is difficult to state detailed 


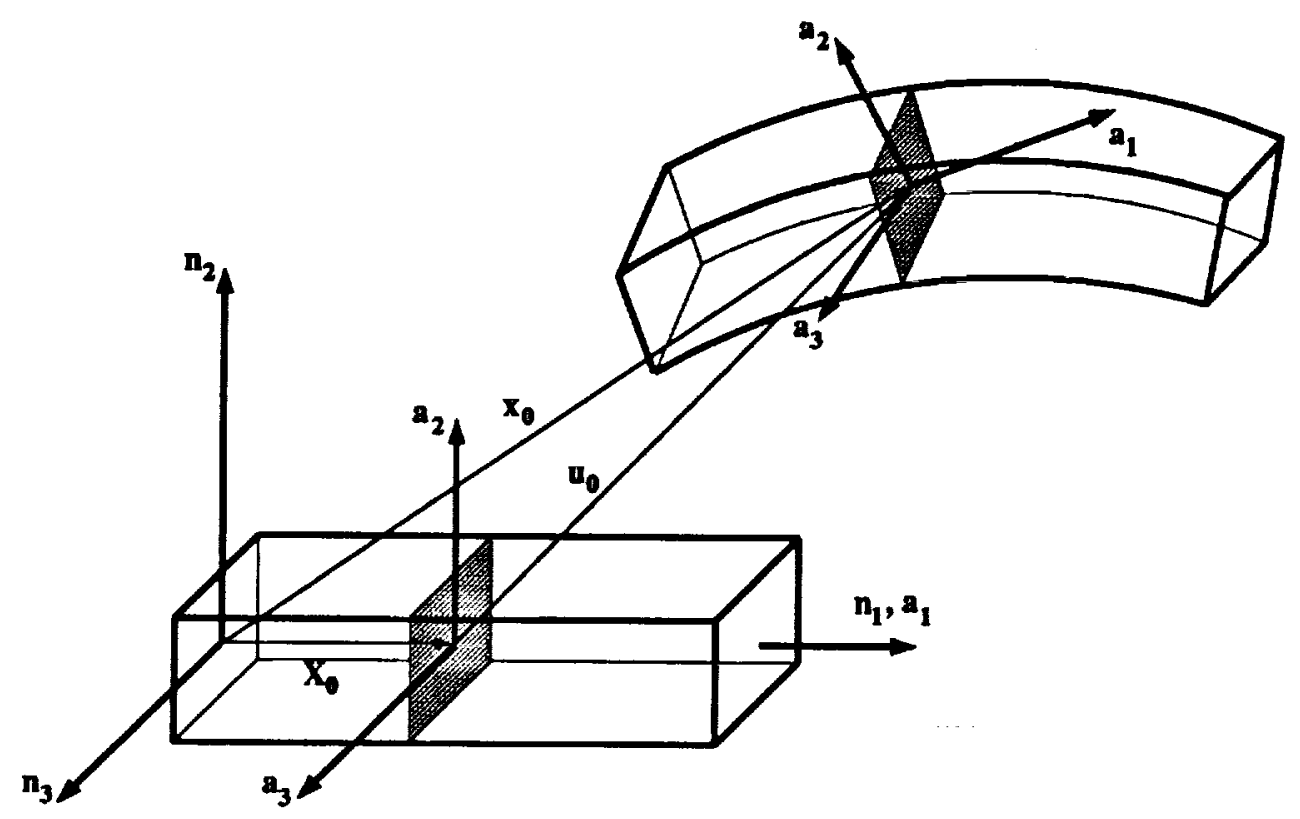

Figure 1: Reference Frames Used to Describe the Beam Kinematics.

rules that encompass all possible situations. Instead the treatment of the $3 \mathrm{D}$ beam element transformations in Sections 9-11 illustrates the basic techniques for "carrying forward" terms such as (21).

\section{KINEMATICS OF BEAM DEFORMATION}

The beam kinematics is based on two assumptions: the TL description, and the Timoshenko beam hypothesis: plane sections remain plane after deformation although not necessarily normal to the deflected longitudinal axis. The beam is isotropically elastic with Young's modulus $E$ and shear modulus $G$. The reference configuration of the beam is straight and prismatic although not necessarily stress free. A local reference frame $\mathbf{n}_{i}$ is attached to it, with $\mathbf{n}_{1}$ directed along the longitudinal axis (the locus of cross section centroids). Axes $\mathbf{n}_{2}$ and $\mathbf{n}_{3}$ are in the plane of the left-end cross section; these will be eventually aligned with the principal inertia axes to simplify some algebraic expressions. Along these axes we attach the coordinate system $\left\{X_{1}, X_{2}, X_{3}\right\}$. This description is schematically shown in Figure 1 .

We further define a set of moving frames, denoted by $\left\{\mathbf{a}_{1}, \mathbf{a}_{2}, \mathbf{a}_{3}\right\}$, parametrized by the longitudinal coordinate $X_{1}$. Initially these frames coincide with $\left\{\mathbf{n}_{1}, \mathbf{n}_{2}, \mathbf{n}_{3}\right\}$, and displace 
rigidly attached to the cross-sections of the moving current configuration, as depicted in the same figure.

A beam particle originally at $\left(X_{1}, X_{2}, X_{3}\right)$ displaces to

$$
\mathbf{x}(\mathbf{X})=\mathbf{x}_{0}\left(X_{1}\right)+\mathbf{R}^{T}\left(X_{1}\right) \zeta\left(X_{2}, X_{3}\right)
$$

where $\mathbf{x}_{0}$ describes the position of the centroid of the given cross-section, $\mathbf{R}$ is a 3 -by-3 orthogonal matrix function that orients the displaced cross section, and $\boldsymbol{\zeta}$ is the cross-section coordinate system $\boldsymbol{\zeta}^{T}=\left[\begin{array}{lll}0 & X_{2} & X_{3}\end{array}\right]$. The displacement field is

$$
\mathbf{u}=\mathbf{x}-\mathbf{X}=\mathbf{u}_{0}+\left(\mathbf{R}^{T}-\mathbf{I}\right) \zeta
$$

where $\mathbf{u}_{0}\left(X_{1}\right)=\mathbf{x}_{0}\left(X_{1}\right)-\mathbf{X}\left(X_{1}\right)$ is the centroidal displacement (see Figure 1).

In what follows $3 \times 3$ skew-symmetric matrices are consistently denoted by placing a tilde over the symbol of their axial 3-vector symbol; for example:

$$
\tilde{\mathbf{a}}=\operatorname{spin}(\mathbf{a})=\left[\begin{array}{ccc}
0 & a_{3} & -a_{2} \\
-a_{3} & 0 & a_{1} \\
a_{2} & -a_{1} & 0
\end{array}\right], \quad \mathbf{a}=\left\{\begin{array}{l}
a_{1} \\
a_{2} \\
a_{3}
\end{array}\right\}=\operatorname{axial}(\tilde{\mathbf{a}}) .
$$

The skew-symmetric curvature matrix $\tilde{\boldsymbol{\kappa}}$ is defined by $\tilde{\boldsymbol{\kappa}}=\mathbf{R}\left(d \mathbf{R}^{T} / d X_{1}\right)$, which is the rate of change of the orthogonal rotation matrix $\mathbf{R}$ with respect to the longitudinal coordinate. The curvature vector is $\kappa=\operatorname{axial}(\tilde{\kappa})$. We shall also require later the variation of angular orientation $\delta \Theta$, defined as the axial vector of the skew matrix $\mathbf{R} \delta \mathbf{R}^{T}$ :

$$
\widetilde{\delta \Theta}=\mathbf{R} \delta \mathbf{R}^{T}=-\delta \mathbf{R} \mathbf{R}^{T}, \quad \delta \Theta=\operatorname{axial}(\widetilde{\delta \Theta})
$$

To define the finite strain measures for the beam, we shall use the displacement gradient matrix

$$
\mathbf{G}=\left[\begin{array}{lll}
g_{11} & g_{12} & g_{13} \\
g_{21} & g_{22} & g_{23} \\
g_{31} & g_{32} & g_{33}
\end{array}\right]=\left[\begin{array}{lll}
\mathbf{g}_{1} & \mathbf{g}_{2} & \mathbf{g}_{3}
\end{array}\right]=\frac{\partial \mathbf{x}}{\partial \mathrm{X}}-\mathbf{I}
$$

where $I$ is the 3 -by-3 identity matrix, and $g_{i}$ are 3-component gradient vectors defined for convenience. The 9-component gradient vector is $\mathbf{g}^{T}=\left(\begin{array}{lll}\mathbf{g}_{1}^{T} & \mathbf{g}_{2}^{T} & \mathbf{g}_{3}^{T}\end{array}\right)$. Next introduce the three unit 3-uples $\mathbf{h}_{j}, j=1,2,3$, such that the $j^{\text {th }}$ term of $\mathbf{h}_{j}$ is equal to one and the other 
two terms are equal to zero. With the help of these quantities, explicit expressions for the displacement gradient vectors $\mathbf{g}$ can be given as

$$
\begin{aligned}
& \mathbf{g}_{1}=\frac{d \mathbf{u}_{0}}{d X_{1}}+\mathbf{R}^{T} \tilde{\kappa} \zeta=\frac{d \mathbf{u}_{0}}{d X_{1}}+\mathbf{R}^{T} \tilde{\zeta}^{T} \kappa, \\
& \mathbf{g}_{2}=\left(\mathbf{R}^{T}-\mathbf{I}\right) \mathbf{h}_{2}, \quad \mathbf{g}_{3}=\left(\mathbf{R}^{T}-\mathbf{I}\right) \mathbf{h}_{3} .
\end{aligned}
$$

The GL finite strain measure is defined as $\frac{1}{2}\left(G+G^{T}+G^{T} G\right)$. The only nonzero components of this tensor are

$$
\begin{array}{ll}
e_{11} & =\quad \mathbf{h}_{1}^{T} \mathbf{g}_{1}+\frac{1}{2} \mathbf{g}_{1}^{T} \mathbf{H g}_{1}, \\
\gamma_{12} & =2 e_{12}=\mathbf{h}_{2}^{T} \mathbf{g}_{1}+\mathbf{h}_{1}^{T} \mathbf{g}_{2}+\mathbf{g}_{1}^{T} \mathbf{H g}_{2}, \\
\gamma_{13} & =2 e_{13}=\mathbf{h}_{3}^{T} \mathbf{g}_{1}+\mathbf{h}_{1}^{T} \mathbf{g}_{3}+\mathbf{g}_{1}^{T} \mathbf{H g}_{3},
\end{array}
$$

where $\mathbf{H}$ is here the $3 \times 3$ identity matrix. By appropriate matrix expansion these strains could be expressed in a form befitting the general expression (9) that involves the 9 -vector $\mathbf{g}$. Observe that the orthogonality of $\mathbf{R}$ gives

$$
\begin{aligned}
e_{22} & =\mathbf{h}_{2}^{T} \mathbf{g}_{2}+\frac{1}{2} \mathbf{g}_{2}^{T} \mathbf{g}_{2}=R_{22}-1+\frac{1}{2}\left(R_{21}^{2}+\left(R_{22}-1\right)^{2}+R_{23}^{2}\right)=R_{22}-1+\frac{1}{2}\left(2-2 R_{22}\right)=0, \\
2 e_{23} & =\mathbf{h}_{2}^{T} \mathbf{g}_{3}+\mathbf{h}_{3}^{T} \mathbf{g}_{2}+\mathbf{g}_{2}^{T} \mathbf{g}_{3}=R_{32}+R_{23}+R_{21} R_{31}+R_{22} R_{32}-R_{32}+R_{23} R_{33}-R_{23}=0,
\end{aligned}
$$

and similarly $e_{33}=0$. This confirms that the only nonzero strains are (28).

The nonzero strains may be rewritten in a more physically suggestive form:

$$
\begin{array}{rlrl}
e_{11} & =e_{b}+e_{f}, & \gamma=\gamma_{12}+\gamma_{13}, \quad \phi=\mathbf{R}\left(\mathbf{h}_{1}+\frac{d \mathbf{u}_{0}}{d X_{1}}\right), \\
e_{b}=\left(\frac{d \mathbf{u}_{0}}{d X_{1}}\right)^{T}\left(\mathbf{h}_{1}+\frac{1}{2} \frac{d \mathbf{u}_{0}}{d X_{1}}\right), & \gamma_{12}=\gamma_{2}+\bar{\gamma}_{2}=\mathbf{h}_{2}^{T} \phi+\mathbf{h}_{2}^{T} \tilde{\zeta}^{T} \kappa, \\
e_{f}=\boldsymbol{\zeta}^{T} \mathcal{K}_{e}+\frac{1}{2} \kappa^{T} \tilde{\zeta} \tilde{\zeta}^{T} \kappa \simeq \boldsymbol{\zeta}^{T} \mathcal{K}_{e}, & \gamma_{13}=\gamma_{3}+\bar{\gamma}_{3}=\mathbf{h}_{3}^{T} \phi+\mathbf{h}_{3}^{T} \tilde{\zeta}^{T} \kappa .
\end{array}
$$

Here $e_{b}, e_{f}$ are stretching and flexural normal strains, $\gamma_{2}$ and $\gamma_{3}$ represent bending-induced shear strains, $\bar{\gamma}_{2}, \bar{\gamma}_{3}$ are torsion-induced shear strains, $\phi$ is the angular distortion vector, and $\mathcal{K}_{e}$ is the effective bending curvature defined as $\mathcal{K}_{e}=\tilde{\phi} \kappa$. The last term in $e_{f}$ represents a squared-curvature contribution to flexure, which can usually be neglected.

From (8) and the fact that $e_{22}=e_{33}=e_{23}=0$, the strain energy stored in the current configuration is

$$
U=\frac{1}{2} \int_{L_{0}} \int_{A_{0}} \mathcal{U} d A_{0} d X_{1}, \quad \text { with } \quad \mathcal{U}=\frac{1}{2}\left[E e_{11}^{2}+G\left(\gamma_{12}^{2}+\gamma_{13}^{2}\right)\right]+s_{11}^{0} e_{11}+s_{12}^{0} \gamma_{12}+s_{13}^{0} \gamma_{13}
$$




\section{CORE BEAM EQUATIONS}

To link up with the general formulation of Sections 4-5, we arrange the nonzero GL strains (28) as $e_{1}=e_{11}, e_{2}=\gamma_{12}$ and $e_{3}=\gamma_{13}$, and the associated PK2 stresses as $s_{1}=s_{11}$, $s_{2}=s_{12}$ and $s_{3}=s_{13}$. The elastic moduli are $E_{11}=E, E_{22}=E_{33}=G$, others zero. The constitutive equations are $s_{11}=s_{11}^{0}+E e_{11}, s_{12}=s_{12}^{0}+G \gamma_{12}$, and $s_{13}=s_{13}^{0}+G \gamma_{13}$. Although the displacement gradient vector $\mathbf{g}$ has 9 components, the formulas that follow are usually expressed in more natural form by using the 3-component subvectors $\mathbf{g}_{i}$ defined in (27).

The spectral core stiffnesses can be compactly expressed in terms of the vectors $\mathbf{c}_{i}=$ $\mathbf{h}_{i}+\frac{1}{2} \mathbf{H g}_{i}$ and $\mathbf{b}_{\mathbf{i}}=\mathbf{h}_{\mathbf{i}}+\mathbf{H g}_{\mathbf{i}}$ for $i=1,2,3$, where no subscript is needed in $\mathbf{H} \equiv \mathbf{I}$. Applying (13) we obtain for the spectral energy stiffness

$$
\mathbf{S}^{U}=\left[\begin{array}{ccc}
E \mathbf{S}_{1}^{U}+G\left(\mathbf{S}_{2}^{U}+\mathbf{S}_{3}^{U}\right) & G \mathbf{S}_{4}^{U} & G \mathbf{S}_{5}^{U} \\
G \mathbf{S}_{4}^{U^{T}} & G \mathbf{S}_{1}^{U} & 0 \\
G \mathbf{S}_{5}^{U^{T}} & 0 & G \mathbf{S}_{1}^{U}
\end{array}\right]+\left[\begin{array}{ccc}
s_{11}^{0} \mathbf{H} & s_{12}^{0} \mathbf{H} & s_{13}^{0} \mathbf{H} \\
s_{12}^{0} \mathbf{H} & 0 & 0 \\
s_{13}^{0} \mathbf{H} & 0 & 0
\end{array}\right]
$$

where $S_{1}^{U}=c_{1} c_{1}^{T}, S_{2}^{U}=c_{2} c_{2}^{T}, S_{3}^{U}=c_{3} c_{3}^{T}, S_{4}^{U}=c_{2} c_{1}^{T}$ and $S_{5}^{U}=c_{3} c_{1}^{T}$. At the force residual level we obtain for $\mathbf{S}^{r}$ a form similar to (32) except that the prestresses $s_{1 j}^{0}, j=1,2,3$ have to be replaced by the midpoint stresses $\frac{1}{2}\left(s_{1 j}^{0}+s_{1 j}\right)$ in the second matrix.

The internal force vector conjugate to $\delta \mathbf{g}$ is $\boldsymbol{\Phi}=\mathbf{S}^{r} \mathbf{g}+\Phi^{0}=\Phi_{\sigma}+\Phi_{r}$, in which

$$
\Phi_{\sigma}=\left\{\begin{array}{c}
s_{11} b_{1} \\
0 \\
0
\end{array}\right\}, \quad \Phi_{r}=\left\{\begin{array}{c}
s_{12} b_{2}+s_{13} b_{3} \\
s_{12} b_{1} \\
s_{13} b_{1}
\end{array}\right\}
$$

represent the contribution of the normal and shear stresses, respectively.

The principal core tangent stiffness matrix $\mathbf{S}=\mathbf{S}_{M}+\mathbf{S}_{G P}$ is obtained from (16). The material stiffness is

$$
\mathbf{S}_{M}=\left[\begin{array}{ccc}
E \mathbf{S}_{1}+G\left(\mathbf{S}_{2}+\mathbf{S}_{3}\right) & G \mathbf{S}_{4} & G \mathbf{S}_{5} \\
G \mathbf{S}_{4}^{T} & G \mathbf{S}_{1} & 0 \\
G \mathbf{S}_{5}^{T} & 0 & G \mathbf{S}_{1}
\end{array}\right]
$$

where $S_{1}=b_{1} b_{1}^{T}, S_{2}=b_{2} b_{2}^{T}, S_{3}=b_{3} b_{3}^{T}, S_{4}=b_{2} b_{1}^{T}$ and $S_{5}=b_{3} b_{1}^{T}$. The principal geometric stiffness is

$$
\mathbf{S}_{G P}=\left[\begin{array}{ccc}
s_{11} \mathbf{H} & s_{12} \mathbf{H} & s_{13} \mathbf{H} \\
s_{12} \mathbf{H} & 0 & 0 \\
s_{13} \mathbf{H} & 0 & 0
\end{array}\right]=\left[\begin{array}{ccc}
\left(s_{11}^{0}+E e_{11}\right) \mathbf{H} & \left(s_{12}^{0}+G \gamma_{12}\right) \mathbf{H} & \left(s_{12}^{0}+G \gamma_{13}\right) \mathbf{H} \\
\left(s_{12}^{0}+G \gamma_{12}\right) \mathbf{H} & 0 & 0 \\
\left(s_{12}^{0}+G \gamma_{13}\right) \mathbf{H} & 0 & 0
\end{array}\right] .
$$


The contribution of $\left(\delta^{2} \mathrm{~g}\right)^{T} \Phi$ to the complementary geometric stiffness depends on the target variables in the ensuing transformation phase described in the next three sections. This phase is carried out in three stages:

1. From particle displacement gradients to generalized gradients $\mathbf{w}$ at each cross section. An integration over the cross section area is involved.

2. From generalized gradients $\mathbf{w}$ to cross-section orientation coordinates $\mathbf{z}$. The rotational parametrization is introduced at this stage.

3. From cross-section orientation coordinates to finite-element nodal degrees of freedom $\mathbf{v}$. An integration over the element length, as defined by the displacement interpolation, is involved.

The first two transformation stages are summarized in Tables 1 and 2, which together also serve to define notation.

\section{TRANSFORMATION TO GENERALIZED GRADIENTS}

The first set of target variables are the generalized gradients $\mathbf{w}\left(X_{1}\right)$ at each reference cross section defined by the longitudinal coordinate $X_{1}$. The components of $w$ are indirectly given through their first variation:

$$
\delta \mathbf{w}=\left\{\begin{array}{lll}
\frac{d \delta \mathbf{u}_{0}}{d X_{1}} & \frac{d \delta \Theta}{d X_{1}} & \delta \Theta
\end{array}\right\}^{T}
$$

where $\delta \Theta$, defined in (25) measures the variation of angular orientation. Because this is not generally integrable for three-dimensional motions, it is not possible to express $\Theta$ as a unique function of the displacements.

The variation of $\mathbf{g}_{1}$ is

$$
\delta \mathbf{g}_{1}=\frac{d \delta \mathbf{u}_{0}}{d X_{1}}+\mathbf{R}^{T} \tilde{\boldsymbol{\zeta}}^{T} \frac{d \delta \Theta}{d X_{1}}+\mathbf{R}^{T} \tilde{\boldsymbol{\zeta}}^{T} \tilde{\boldsymbol{\kappa}} \delta \Theta+\mathbf{R}^{T} \widetilde{\delta \Theta} \tilde{\zeta}^{T} \boldsymbol{\kappa}
$$

where we used the relation ${ }^{26} \delta \boldsymbol{\kappa}=d \delta \Theta / d X_{1}+\tilde{\boldsymbol{\kappa}} \delta \Theta$. On using the commutative law $\tilde{\mathbf{a} b}=\tilde{\mathbf{b}}^{T} \mathbf{a}$ and Jacobi's identity $\widetilde{\mathbf{a} b}=\tilde{\mathbf{a}} \tilde{\mathbf{b}}-\tilde{\mathbf{b}} \tilde{\mathbf{a}}$ we may rewrite (37) as

$$
\delta \mathbf{g}_{1}=\frac{d \delta \mathbf{u}_{0}}{d X_{1}}+\mathbf{R}^{T} \tilde{\zeta}^{T} \frac{d \delta \Theta}{d X_{1}}+\mathbf{R}^{T} \tilde{\boldsymbol{\kappa}}^{T} \tilde{\zeta} \delta \Theta
$$

For the other gradient vectors we have $\delta \mathbf{g}_{2}=\delta \mathbf{R}^{T} \mathbf{h}_{2}=\mathbf{R}^{T} \widetilde{\delta \Theta} \mathbf{h}_{2}=\mathbf{R}^{T} \tilde{\mathbf{h}}_{2}^{T} \delta \Theta$ and $\delta \mathbf{g}_{3}=$ 


\begin{tabular}{|c|c|c|c|c|}
\hline 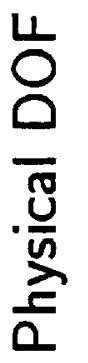 & 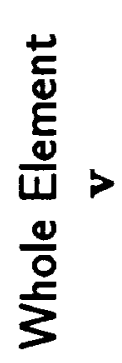 & | & $\begin{array}{c}4-1 \\
3 \\
0 \\
11 \\
D \\
0\end{array}$ & $\begin{array}{l}b \\
0 \\
1 \\
3 \\
3 \\
11 \\
11 \\
D \\
\infty \\
\infty\end{array}$ \\
\hline 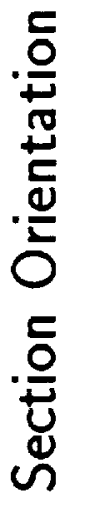 & 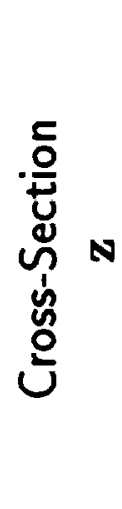 & | & 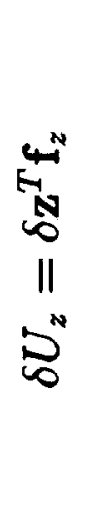 & 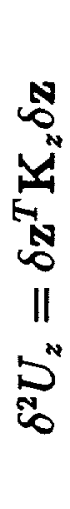 \\
\hline 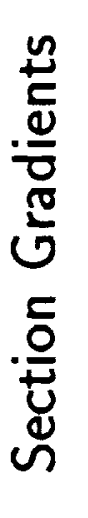 & 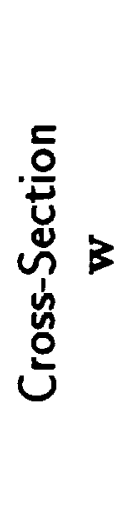 & 1 & $\begin{array}{c}R \\
F \\
B \\
11 \\
11 \\
0 \\
0 \\
0\end{array}$ & 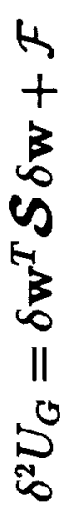 \\
\hline 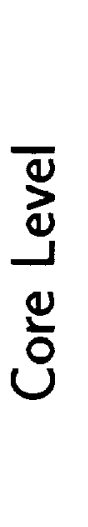 & 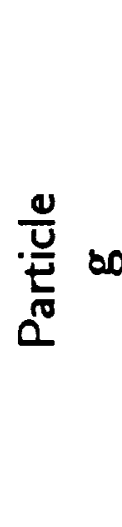 & 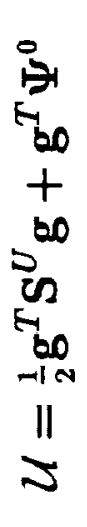 & 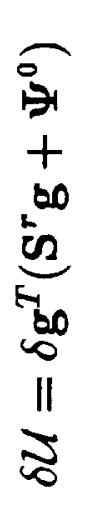 & 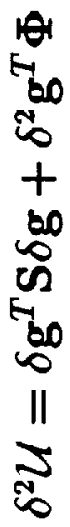 \\
\hline
\end{tabular}

告

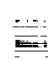

늘

$\bar{\equiv}$ 
$\mathbf{R}^{T} \tilde{\mathbf{h}}_{3}^{T} \delta \Theta$. These relations can be collected in matrix form as

$$
\delta \mathbf{g}=\left\{\begin{array}{c}
\delta \mathbf{g}_{1} \\
\delta \mathbf{g}_{2} \\
\delta \mathbf{g}_{3}
\end{array}\right\}=\left[\begin{array}{ccc}
\mathbf{I} & \mathbf{R}^{T} \tilde{\boldsymbol{\zeta}}^{T} & \mathbf{R}^{T} \tilde{\boldsymbol{\kappa}}^{T} \tilde{\boldsymbol{\zeta}} \\
0 & 0 & \mathbf{R}^{T} \tilde{\mathbf{h}}_{2}^{T} \\
0 & 0 & \mathbf{R}^{T} \tilde{\mathbf{h}}_{3}^{T}
\end{array}\right]\left\{\begin{array}{c}
\frac{d \delta \mathbf{u}_{0}}{d X_{1}} \\
\frac{d \delta \Theta}{d X_{1}} \\
\delta \Theta
\end{array}\right\}=\left[\begin{array}{c}
\mathbf{W}_{1} \\
\mathbf{W}_{2} \\
\mathbf{W}_{3}
\end{array}\right] \delta \mathbf{w}=\mathbf{W} \delta \mathbf{w}
$$

where $I$ is the 3 -by-3 identity matrix and $W_{i}$ are 3 -by- 9 matrices. The second variation of $g$, which is required for the complementary stiffness, is

$$
\begin{aligned}
\delta^{2} \mathbf{g}_{1}= & \mathbf{R}^{T} \widetilde{\delta \Theta} \tilde{\zeta}^{T} \frac{d \delta \Theta}{d X_{1}}+\mathbf{R}^{T} \widetilde{\delta \Theta} \tilde{\zeta}^{T} \tilde{\boldsymbol{\kappa}} \delta \Theta+\frac{d \delta \Theta^{T}}{d X_{1}} \tilde{\boldsymbol{\zeta}}^{T} \widetilde{\delta \Theta} \mathbf{R} \\
& +\delta \Theta^{T} \tilde{\boldsymbol{\kappa}} \tilde{\boldsymbol{\zeta}} \widetilde{\Theta} \mathbf{R}+\delta^{2} \mathbf{R}^{T} \tilde{\boldsymbol{\zeta}}^{T} \boldsymbol{\kappa}+\mathbf{R}^{T} \tilde{\boldsymbol{\zeta}}^{T} \delta^{2} \boldsymbol{\kappa} \\
\delta^{2} \mathbf{g}_{2}= & \delta^{2} \mathbf{R}^{T} \mathbf{i}_{2}, \quad \delta^{2} \mathbf{g}_{3}=\delta^{2} \mathbf{R}^{T} \mathbf{i}_{3}
\end{aligned}
$$

At this point it is appropriate to introduce the following section resultants:

$$
\begin{array}{rlrl}
\mathcal{P} & =A \sigma_{b}+\mathcal{P}^{0}, & & s_{b}=E e_{b}, \\
\mathcal{Q} & =\beta_{1} A \tau+\mathcal{Q}^{0}, & \tau=\tau_{2}+\tau_{3}, \quad \tau_{2}=G \gamma_{2} \mathbf{h}_{2}, \quad \tau_{3}=G \gamma_{3} \mathbf{h}_{3}, \\
\mathcal{M}_{\sigma} & =E \mathbf{I}_{S} \mathcal{K}_{e}+\mathcal{M}_{\sigma}^{0}, & & \mathbf{I}_{S}=\int_{A} \zeta \zeta^{T} d A, \quad \boldsymbol{\kappa}_{e}=\tilde{\phi} \kappa, \\
\mathcal{M}_{\tau} & =\beta_{2} G \mathbf{I}_{P} \boldsymbol{\kappa}+\mathcal{M}_{\tau}^{0}, & \mathbf{I}_{P}=\tilde{\mathbf{h}}_{2}^{T} \mathbf{I}_{S} \tilde{\mathbf{h}}_{2}+\tilde{\mathbf{h}}_{3}^{T} \mathrm{I}_{S} \tilde{\mathbf{h}}_{3} .
\end{array}
$$

Here $\mathcal{P}, \mathcal{Q}, \mathcal{M}_{\sigma}$ and $\mathcal{M}_{\tau}$ are axial forces, transverse shear forces, bending moments and torsional moments, respectively, at the current configuration $\mathcal{C} ; \mathcal{P}^{0}, \mathcal{Q}^{0}, \mathcal{M}_{\sigma}^{0}$ and $\mathcal{M}_{\tau}^{0}$ are similar quantities at the reference configuration $\mathcal{C}_{0} ; \beta_{1}$ and $\beta_{2}$ are transverse-shear and torsion coefficients, respectively, that compensate for the actual shear stress distributions; and $\mathbf{I}_{S}$ and $\mathbf{I}_{P}$ are the cartesian and polar inertia tensors, respectively, of the cross section. Should the axes $X_{2}$ and $X_{3}$ be aligned with the principal inertia axes the latter simplify to

$$
\mathbf{I}_{S}=\left[\begin{array}{ccc}
0 & 0 & 0 \\
0 & I_{22} & 0 \\
0 & 0 & I_{33}
\end{array}\right], \quad \mathbf{I}_{P}=\left[\begin{array}{ccc}
I_{22}+I_{33} & 0 & 0 \\
0 & 0 & 0 \\
0 & 0 & 0
\end{array}\right]
$$

Because the relation between $g$ and $\mathbf{w}$ is of differential type the applicable transformation rules are those of Case III in Section 6, and no energy or secant stiffness survives. Thus only the internal force vector $\mathcal{R}$ and tangent stiffness $\mathcal{S}$ associated with w are derived below. 


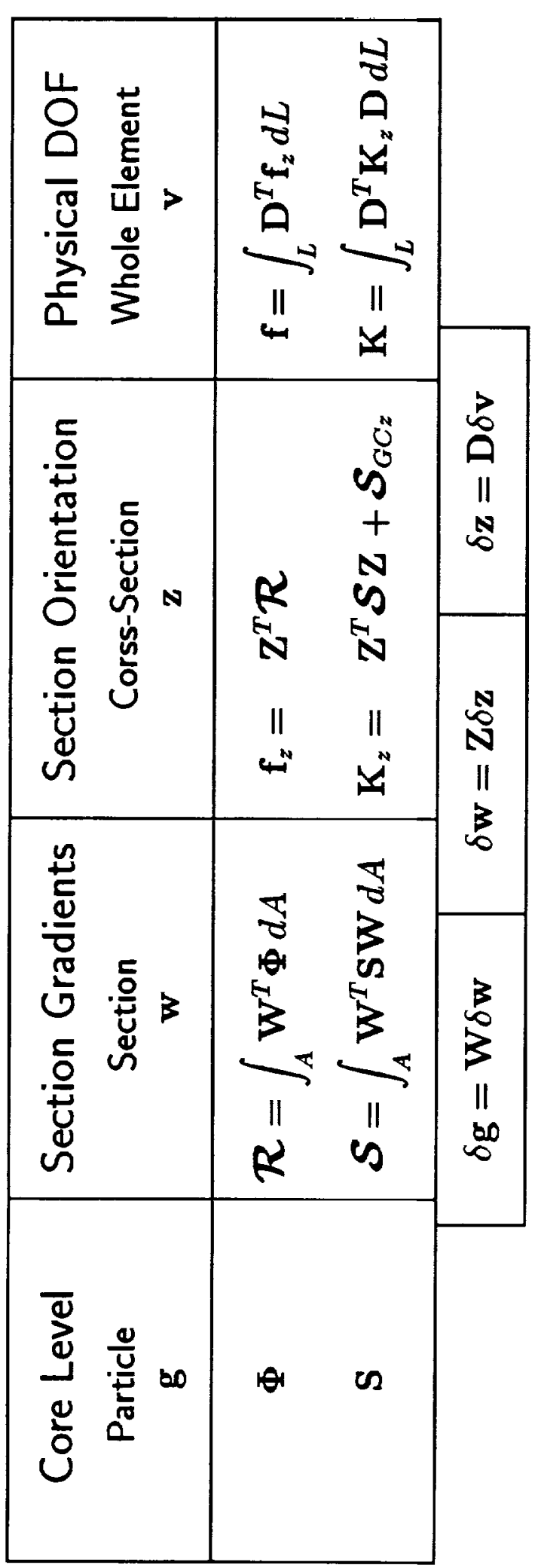



$\bar{E}$

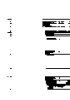

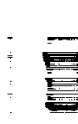

$=$

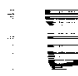

1

$\underset{P}{\cdots}$

2

$\stackrel{=1}{=0}$

㝵

를

$\equiv$
$\equiv$

急

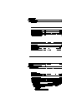

瑟 


\section{Internal Force Vector}

The generalized internal force vector is

$$
\mathcal{R}=\int_{A} \mathbf{W}^{T} \Phi d A=\sum_{i} \int_{A} s_{i} \mathbf{W}^{T} \mathbf{b}_{i} d A=\mathcal{R}_{\sigma}+\mathcal{R}_{\tau}
$$

where $\mathcal{R}_{\sigma}$ and $\mathcal{R}_{\boldsymbol{\tau}}$ are the contributions of the normal and shear stresses respectively. Detailed calculations ${ }^{26}$ result in the following expressions:

$$
\mathcal{R}_{\sigma}=\left[\begin{array}{c}
\mathbf{R}^{T}\left(\mathcal{P} \phi+\tilde{\kappa} \mathcal{M}_{\sigma}\right) \\
\tilde{\phi}^{T} \mathcal{M}_{\sigma} \\
\tilde{\mathcal{K}}_{e}^{T} \mathcal{M}_{\sigma}
\end{array}\right], \quad \mathcal{R}_{\tau}=\left[\begin{array}{c}
\mathbf{R}^{T} \mathcal{Q} \\
\mathcal{M}_{\tau} \\
\tilde{\phi}^{T} \mathcal{Q}+\tilde{\kappa}^{T} \mathcal{M}_{\tau}
\end{array}\right]
$$

Observe that the term $\mathcal{P} \mathbf{R}^{T} \phi$ corresponds to the internal force obtained for a Total Lagrangian truss element. ${ }^{4}$ In (44) and in the sequel we neglect the squared-curvature contribution to flexure, consistent with the approximation introduced in equation (30).

For small deformations we have $\mathbf{R} \approx \mathbf{I}, \tilde{\phi} \approx \tilde{\mathbf{h}}_{1}, \mathcal{K}_{e} \approx \kappa$ and $\tilde{\kappa} \mathcal{M}_{\sigma} \approx 0$. If these approximations are made,

$$
\mathcal{R}_{\sigma}=\left[\begin{array}{c}
\mathcal{P} \mathbf{h}_{1} \\
\tilde{\mathbf{h}}_{1}^{T} \mathcal{M}_{\sigma} \\
0
\end{array}\right], \quad \mathcal{R}_{\tau}=\left[\begin{array}{c}
\mathcal{Q} \\
\mathcal{M}_{\tau} \\
\widetilde{\mathbf{h}}_{1}^{T} \mathcal{Q}
\end{array}\right]
$$

These expressions resemble the classic linearized theory equations. ${ }^{26}$

\section{Tangent Stiffness}

For the tangent stiffness we have the decomposition introduced in Section 6:

$$
\mathcal{S}=\mathcal{S}_{M}+\mathcal{S}_{G P}+\mathcal{S}_{G C}
$$

Furthermore, since $\mathbf{w}$ is nonlinear in downstream variables, the complementary geometric stiffness splits into two components:

$$
\mathcal{S}_{G C}=\mathcal{S}_{G C w}+\mathcal{S}_{G C z},
$$

where $\mathcal{S}_{G C w}$ and $\mathcal{S}_{G C z}$ contains terms that depend on the first and second variations, respectively, of $\mathbf{R}$ and $\boldsymbol{\kappa}$. The notation is suggested by the fact that $\mathcal{S}_{G C w}$ can be merged into $\mathcal{S}_{G P}$ 
to yield the geometric stiffness $\mathcal{S}_{G w}=\mathcal{S}_{G P}+\mathcal{S}_{G C w}$, which is associated with the generalized gradients $w$ and independent of the rotational parametrization selected in the next set of target variables $\mathbf{z}$. On the other hand, the kernel $\mathcal{S}_{G C z}$ cannot be extracted at the $\mathrm{w}$ level and must be carried forward to the $\mathrm{z}$ level because it is parametrization dependent.

Each of the components in (46)-(47) may be expressed as the sum of two contributions, one from the normal stresses and one from the shear stresses:

$$
\mathcal{S}_{M}=\mathcal{S}_{M \sigma}+\mathcal{S}_{M \tau}, \quad \mathcal{S}_{G P}=\mathcal{S}_{G P \sigma}+\mathcal{S}_{G P \tau}, \quad \mathcal{S}_{G C x}=\mathcal{S}_{G C x \sigma}+\mathcal{S}_{G C x \tau}, \quad x=w, z .
$$

\section{Material Stiffness}

The generalized core material stiffness is given by the congruential transformation

$$
\mathcal{S}_{M}=\int_{A} \mathbf{W}^{T} \mathbf{S}_{M} \mathbf{W} d A=\sum_{i} \int_{A} E_{i} \mathbf{W}^{T} \mathbf{b}_{i} \mathbf{b}_{i}^{T} \mathbf{W} d A=\mathcal{S}_{M \sigma}+\mathcal{S}_{M \tau} .
$$

Carrying out the algebraic manipulations one obtains ${ }^{26}$

$$
\begin{aligned}
& \mathcal{S}_{M \sigma}=E\left[\begin{array}{ccc}
\mathbf{R}^{T}\left(A \phi \phi^{T}+\tilde{\boldsymbol{\kappa}}^{T} \mathbf{I}_{S} \tilde{\boldsymbol{\kappa}}\right) \mathbf{R} & \mathbf{R}^{T} \tilde{\boldsymbol{\kappa}} \mathbf{I}_{S} \tilde{\boldsymbol{\phi}} & \mathbf{R}^{T} \tilde{\boldsymbol{\kappa}} \mathbf{I}_{S} \tilde{\mathcal{K}}_{e} \\
& \tilde{\phi}^{T} \mathbf{I}_{S} \tilde{\phi} & \tilde{\phi}^{T} \mathbf{I}_{S} \tilde{\mathcal{K}}_{e} \\
& & \tilde{\mathcal{K}}_{e}^{T} \mathbf{I}_{S} \tilde{\mathcal{K}}_{e}
\end{array}\right], \\
& \mathcal{S}_{M \tau}=\alpha G\left[\begin{array}{ccc}
A \mathbf{R}^{T} \mathbf{I}_{\perp} \mathbf{R} & \mathbf{0} & A \mathbf{R}^{T} \mathbf{I}_{\perp} \tilde{\boldsymbol{\phi}} \\
& \mathbf{I}_{P} & \mathbf{I}_{P} \tilde{\boldsymbol{\kappa}} \\
s y m m & & A \tilde{\phi}^{T} \mathbf{I}_{\perp} \tilde{\boldsymbol{\phi}}+\tilde{\boldsymbol{\kappa}}^{T} \mathbf{I}_{P} \tilde{\boldsymbol{\kappa}}
\end{array}\right], \quad \text { in which } \mathbf{I}_{\perp}=\left[\begin{array}{lll}
0 & 0 & 0 \\
0 & 1 & 0 \\
0 & 0 & 1
\end{array}\right] \text {. }
\end{aligned}
$$

Observe that the contribution $\mathbf{R}^{T} \phi \phi^{T} \mathbf{R}$ is the core material stiffness of a Total-Lagrangian bar element. ${ }^{4}$

\section{Geometric Stiffness due to Normal Stresses}

It is convenient to work out together all geometric stiffness terms produced by the normal stresses, i.e.

$$
\mathcal{S}_{G \sigma}=\mathcal{S}_{G P \sigma}+\mathcal{S}_{G C w \sigma}+\mathcal{S}_{G C z \sigma}=\mathcal{S}_{G w \sigma}+\mathcal{S}_{G C z \sigma} .
$$

The appropriate definitions are

$$
\begin{aligned}
& \mathcal{S}_{G P \sigma}=\int_{A} s_{11} \mathbf{W}_{1}^{T} \mathbf{H} \mathbf{W}_{1} d A \\
& \mathcal{S}_{G C \sigma}=\int_{A} s_{11} \mathbf{b}_{1} \delta^{2} \mathbf{g} d A=\delta \mathbf{w}^{T} \mathcal{S}_{G C w \sigma} \delta \mathbf{w}+\mathcal{F}\left(\delta^{2} \mathbf{R}, \delta^{2} \kappa\right),
\end{aligned}
$$


where $\mathcal{F}$ contains $\mathcal{S}_{G C z}$ as $\mathrm{z}$ level kernel. Carrying out the algebraic manipulations one obtains $^{26}$

$$
\mathcal{S}_{G w \sigma}=\mathcal{S}_{G P \sigma}+\mathcal{S}_{G C w \sigma}=\left[\begin{array}{ccc}
\mathcal{P I} & \mathbf{R}^{T} \widetilde{\mathcal{M}}_{\sigma}^{T} & \mathbf{R}^{T} \tilde{\boldsymbol{\kappa}}^{T} \widetilde{\mathcal{M}}_{\sigma} \\
& 0 & \widetilde{\mathcal{M}}_{\sigma} \tilde{\phi} \\
s y m m & & \tilde{\phi} \widetilde{\mathcal{M}}_{\sigma} \tilde{\kappa}+\tilde{\kappa}^{T} \widetilde{\mathcal{M}}_{\sigma} \tilde{\phi}
\end{array}\right]
$$

The term $\mathcal{P}$ I corresponds to the well known generalized core geometric stiffness of the Total Lagrangian bar element. ${ }^{4}$

The higher order term in (53) may be expressed as

$$
\mathcal{F}_{\sigma}\left(\delta^{2} \mathbf{R}, \delta^{2} \boldsymbol{\kappa}\right)=\mathcal{M}_{\sigma}^{T} \tilde{\phi} \delta^{2} \kappa+\phi^{T} \mathbf{R} \delta^{2} \mathbf{R}^{T} \tilde{\kappa} \mathcal{M}_{\sigma}=\delta \mathbf{z}^{T}\left(\mathbf{V}\left(\tilde{\phi}^{T} \mathcal{M}_{\sigma}\right)+\mathrm{U}\left(\tilde{\kappa} \mathcal{M}_{\sigma} ; \phi\right)\right) \delta \mathbf{z}
$$

Thus

$$
\mathcal{S}_{G C z \sigma}=\mathrm{V}\left(\tilde{\phi}^{T} \mathcal{M}_{\sigma}\right)+\mathrm{U}\left(\tilde{\boldsymbol{\kappa}} \mathcal{M}_{\sigma} ; \phi\right)
$$

Since the next-level target variables $\mathbf{z}$ include the finite rotation parametrization, matrices $\mathbf{V}$ and $U$ depend on that choice. They are the source of unsymmetries in the stiffness matrices when certain rotational parametrizations are adopted, such as the incremental rotation vector. For the rotational vector defined in (63), these matrices are symmetric.

\section{Geometric Stiffness due to Shear Stresses}

The contribution of the shear stresses to the geometric stiffness is

$$
\mathcal{S}_{G \tau}=\mathcal{S}_{G P \tau}+\mathcal{S}_{G C w \tau}+\mathcal{S}_{G C z \tau}=\mathcal{S}_{G w \tau}+\mathcal{S}_{G C z \tau}
$$

The appropriate definitions are

$$
\begin{aligned}
& \mathcal{S}_{G P \tau}=\int_{A} s_{12}\left(\mathbf{W}_{1}^{T} \mathbf{H W}_{2}+\mathbf{W}_{2} \mathbf{H W}_{1}\right)+s_{13}\left(\mathbf{W}_{1}^{T} \mathbf{H} \mathbf{W}_{3}+\mathbf{W}_{3} \mathbf{H W} \mathbf{W}_{1}\right) d A \\
& \mathcal{S}_{G C \tau}=\int_{A}\left(s_{12} \mathbf{b}_{2}+s_{13} \mathbf{b}_{3}\right) \delta^{2} \mathbf{g} d A=\delta \mathbf{w}^{T} \mathcal{S}_{G C w \tau} \delta \mathbf{w}+\mathcal{F}_{\tau}\left(\delta^{2} \mathbf{R}, \delta^{2} \boldsymbol{\kappa}\right) .
\end{aligned}
$$

Carrying out manipulations one obtains the surprisingly simple form for $\mathcal{S}_{G w \tau}$

$$
\mathcal{S}_{G w \tau}=\mathcal{S}_{G P \tau}+\mathcal{S}_{G C w \tau}=\left[\begin{array}{ccc}
0 & \mathbf{0} & \mathbf{R}^{T} \tilde{\mathcal{Q}}^{T} \\
& \mathbf{0} & \mathbf{0} \\
s y m m & & \mathbf{0}
\end{array}\right]
$$


The terms due to the second variation of $\mathbf{g}$ become

$$
\mathcal{F}_{\tau}=\mathcal{Q}^{T} \delta^{2} \mathbf{R} \Phi+\mathcal{M}_{\tau}^{T} \delta^{2} \kappa
$$

The stiffness kernel carried forward to the $\mathrm{z}$ level is

$$
\mathcal{S}_{G C \boldsymbol{z} \tau}=\mathrm{V}\left(\mathcal{M}_{\tau}\right)+\mathrm{U}(\mathcal{Q} ; \boldsymbol{\Phi})
$$

\section{TRANSFORMATION TO THE ROTATIONAL VECTOR}

The next transformation stage passes from $\mathrm{w}$ to $\mathrm{z}$, which is a 9-vector of generalized displacements, also associated with a beam section, which embodies the parametrization of the cross section rotation:

$$
\mathbf{z}=\left\{\begin{array}{lll}
\frac{d \mathbf{u}_{0}}{d X_{1}} & \frac{d \alpha}{d X_{1}} & \alpha
\end{array}\right\}^{T}, \quad \delta \mathbf{z}=\left\{\begin{array}{lll}
\frac{d \delta \mathbf{u}_{0}}{d X_{1}} & \frac{d \delta \alpha}{d X_{1}} & \delta \alpha
\end{array}\right\}^{T} .
$$

Here $\alpha$ denotes the rotational vector parametrization defined by the standard formulas

$$
\boldsymbol{\alpha}=\operatorname{axial} \tilde{\boldsymbol{\alpha}}, \quad \mathbf{R}=\exp \left(\tilde{\boldsymbol{\alpha}}^{T}\right),
$$

and which may be extracted from $\mathbf{R}$ by

$$
\tilde{\boldsymbol{\alpha}}=\log \mathbf{R}=\frac{\arcsin (\tau)}{2 \tau} \operatorname{axial}\left(\mathbf{R}^{T}-\mathbf{R}\right), \quad \tau=\frac{1}{2}\left\|\operatorname{axial}\left(\mathbf{R}^{T}-\mathbf{R}\right)\right\| .
$$

Because only the variations of $w$ are known the relation between $w$ and $z$ is also of differential type:

$$
\delta \mathbf{w}=\mathbf{Z} \delta \mathbf{z}, \quad \text { or } \delta \mathbf{w}=\left[\begin{array}{ccc}
\mathbf{I} & 0 & 0 \\
0 & \mathbf{Y}(\boldsymbol{\alpha}) & \frac{d \mathbf{Y}(\boldsymbol{\alpha})}{d X_{1}} \\
0 & 0 & \mathbf{Y}(\boldsymbol{\alpha})
\end{array}\right]\left\{\begin{array}{c}
\frac{d \delta \mathbf{u}_{0}}{d X_{1}} \\
\frac{d \delta \boldsymbol{\alpha}}{d X_{1}} \\
\delta \boldsymbol{\alpha}
\end{array}\right\}
$$

in which

$$
\mathbf{Y}(\boldsymbol{\alpha})=\frac{\sin |\boldsymbol{\alpha}|}{|\boldsymbol{\alpha}|} \mathbf{I}+\left(1-\frac{\sin |\boldsymbol{\alpha}|}{|\boldsymbol{\alpha}|}\right) \frac{\boldsymbol{\alpha} \boldsymbol{\alpha}^{T}}{|\boldsymbol{\alpha}|^{2}}-\frac{1-\cos |\boldsymbol{\alpha}|}{|\alpha|^{2}} \tilde{\alpha} .
$$

Applying the transformations (65) we find for the internal force and the material and principal-geometric components of the tangent stiffness matrix:

$$
\mathbf{f}_{z}=\mathbf{Z}^{T}\left(\mathcal{R}_{\sigma}+\mathcal{R}_{\tau}\right), \quad \mathbf{K}_{M z}=\mathbf{Z}^{T}\left(\mathcal{S}_{M}\right) \mathbf{Z}, \quad \mathbf{K}_{G P z}=\mathbf{Z}^{T}\left(\mathcal{S}_{G w}\right) \mathbf{Z} .
$$


The materialization of the geometric stiffness terms $\mathcal{S}_{G C_{z \sigma} \sigma}$ and $\mathcal{S}_{G C z \tau}$ for the rotational vector is more complex. We state here only the final result: for $\mathcal{F}_{\tau}$

$$
\mathbf{U}(\mathbf{Q} ; \boldsymbol{\Phi})=\left[\begin{array}{ccc}
0 & 0 & 0 \\
& 0 & 0 \\
\text { symm } & & \mathbf{U}^{\tau}
\end{array}\right], \quad \mathbf{V}\left(\mathcal{M}_{\tau}\right)=\left[\begin{array}{ccc}
0 & 0 & 0 \\
& 0 & \mathbf{V}_{1}^{\tau} \\
\text { symm } & & \mathbf{V}_{2}^{\tau}
\end{array}\right]
$$

where

$$
\begin{aligned}
& \mathbf{U}^{T}=c_{1} \mathbf{Q}^{T} \mathbf{\Phi} \mathbf{I}+c_{2}\left(\mathbf{Q} \Phi^{T}+\boldsymbol{\Phi}^{T} \mathbf{Q}\right)+c_{3}\left(\mathbf{Q}^{T} \boldsymbol{\Phi} \boldsymbol{\alpha} \boldsymbol{\alpha}^{T}+\mathbf{Q}^{T} \tilde{\boldsymbol{\alpha}} \mathbf{\Phi} \mathbf{I}+\tilde{\mathbf{Q}}^{T} \boldsymbol{\Phi} \boldsymbol{\alpha}^{T}+\alpha \boldsymbol{\Phi}^{T} \tilde{\mathbf{Q}}\right)+ \\
& c_{5}\left(\alpha^{T} \Phi\left(\mathbf{Q} \alpha^{T}+\alpha \mathbf{Q}^{T}\right)+\alpha^{T} \mathbf{Q}\left(\alpha \Phi^{T}+\Phi \alpha^{T}\right)+\mathbf{Q}^{T} \alpha \alpha^{T} \Phi \mathbf{I}\right)+ \\
& c_{4} \mathbf{Q}^{T} \tilde{\alpha} \Phi \alpha \alpha^{T}+c_{6} \mathbf{Q}^{T} \alpha \alpha^{T} \Phi \alpha \alpha^{T} \\
& \mathrm{~V}_{1}^{\tau}=c_{2} \widetilde{\mathcal{M}}_{\tau}^{T}+c_{3} \alpha \mathcal{M}_{\tau}^{T}+c_{5} \alpha \alpha^{T} \widetilde{\mathcal{M}}_{\tau}+c_{7}\left(\mathcal{M}_{\tau} \alpha^{T}+\alpha^{T} \mathcal{M}_{\tau} \mathrm{I}\right)+c_{8} \alpha^{T} \mathcal{M}_{\tau} \alpha \alpha^{T} \\
& \mathrm{~V}_{2}^{\tau}=-c_{3}{\frac{d \alpha^{T}}{d X_{1}}}^{T} \mathcal{M}_{\tau} \mathrm{I}-c_{4}{\frac{d \alpha^{T}}{d X_{1}}}^{T} \mathcal{M}_{\tau} \alpha \alpha^{T}+ \\
& c_{5}\left(\frac{\widetilde{d \alpha}^{T}}{d X_{1}} \mathcal{M}_{\tau} \alpha^{T}+\alpha \mathcal{M}_{\tau}^{T} \frac{\widetilde{d \alpha}}{d X_{1}}+\alpha^{T} \frac{\widetilde{d \alpha}}{d X_{1}} \mathcal{M}_{\tau} \mathrm{I}\right)+ \\
& c_{6} \boldsymbol{\alpha}^{T} \frac{\widetilde{d \alpha}}{d X_{1}} \mathcal{M}_{\tau} \alpha \alpha^{T}+c_{7}\left(\frac{d \alpha}{d X_{1}} \mathcal{M}_{\tau}^{T}+\mathcal{M}_{\tau} \frac{d \alpha^{T}}{d X_{1}}\right)+ \\
& c_{8}{\frac{d \alpha^{T}}{d X_{1}}}^{T} \alpha\left(\alpha \mathcal{M}_{\tau}^{T}+\mathcal{M}_{\tau} \alpha^{T}+\alpha^{T} \mathcal{M}_{\tau} \mathbf{I}\right)+c_{9}{\frac{d \alpha^{T}}{d X_{1}}}^{T} \alpha \alpha^{T} \mathcal{M}_{\tau} \alpha \alpha^{T}
\end{aligned}
$$

in which

$$
\begin{array}{lll}
c_{1}=-\frac{\sin \alpha}{\alpha}, & c_{2}=\frac{1-\cos \alpha}{\alpha^{2}}, & c_{3}=\frac{\sin \alpha-\alpha \cos \alpha}{\alpha^{3}}, \\
c_{4}=-\frac{c_{1}+3 c_{3}}{\alpha^{2}}, & c_{5}=-\frac{c_{1}+2 c_{2}}{\alpha^{2}}, & c_{6}=-\frac{c_{3}+4 c_{5}}{\alpha^{2}}, \\
c_{7}=\frac{1+c_{1}}{\alpha^{2}}, & c_{8}=\frac{3 c_{3}-2 c_{2}}{\alpha^{2}}, & c_{9}=\frac{c_{5}-5 c_{8}}{\alpha^{2}} .
\end{array}
$$

A similar approach can be taken with (56), which defines $\mathcal{F}_{\sigma}$. The tangent stiffness matrix can be obtained by superposing all contributions.

\section{TRANSFORMATION TO FINITE ELEMENT FREEDOMS}

The final stage introduces a finite element representation for the degrees of freedom. The beam or beam assembly is divided into a set of two-node finite elements. Each of these nodes has 
three displacement degrees of freedom and three rotational degrees of freedom corresponding to the three $\left(X_{1}, X_{2}, X_{3}\right)$ components of the rotational vector $\alpha$. Each element in turn has twelve freedoms which are collected in the array $v^{T}=\left\{\begin{array}{ll}d_{n} & \alpha_{n}\end{array}\right\}^{T}$ where $d_{n}$ collects the six translational freedoms while $\alpha_{n}$ collects the six rotations. The cross-section state vector $\mathrm{z}$ is approximated inside each element by

$$
\mathrm{z}=\left[\begin{array}{cc}
\mathrm{N} & 0 \\
0 & \frac{d \mathbf{N}}{d X_{1}} \\
0 & \mathrm{~N}
\end{array}\right]\left\{\begin{array}{l}
\mathrm{d}_{n} \\
\alpha_{n}
\end{array}\right\}=\mathrm{D}\left\{\begin{array}{c}
\mathrm{d}_{n} \\
\alpha_{n}
\end{array}\right\}=\mathrm{Dv},
$$

where $\mathbf{N}$ is a matrix of linear shape functions. Since $\delta \mathbf{z}=\mathbf{D} \delta \mathbf{v}$ the final internal force vector $f$ and tangent stiffness matrix $K$ of each element are obtained as

$$
\mathbf{f}=\int_{L} \mathbf{D}^{T} \mathbf{f}_{z} d L, \quad \mathbf{K}=\int_{L} \mathbf{D}^{T} \mathbf{K}_{z} \mathbf{D} d L
$$

where $L$ is the element length.

The choice of shape functions for the rotational vector poses some subtle questions. In small-deflection analysis it is common practice to select all Timoshenko beam shape functions to be linear in $X_{1}$. This choice obviously enforces nodal compatibility while preserving constant curvature states. But for finite deflections a linear interpolation for the rotational vector components cannot exactly represent a constant curvature state unless the rotations are about a single axis (plane rotations). The same is true if the rotation matrix $\mathbf{R}\left(X_{1}\right)$ is interpolated linearly. On the other hand, linear interpolation of Euler parameters does preserve the constant curvature state. This motivated a development of an interpolation scheme that starts from the 4 Euler parameters $\epsilon_{i}\left(X_{1}\right), i=0,1,2,3, \sum_{i} \epsilon_{i}^{2}=1$ that orient the normal of a cross section at $X_{1}$. These are collected in the 4-vector $\epsilon=\left\{\begin{array}{llll}\epsilon_{0} & \epsilon_{1} & \epsilon_{2} & \epsilon_{3}\end{array}\right\}^{T}$. Given the eight end values $\epsilon(0)$ and $\epsilon(L)$ the interpolation that can copy a constant curvature vector $\kappa$ is found to be $\mathrm{e}^{26}$

$$
\epsilon(\zeta)=\cos (\zeta)\left(1-\frac{\tan (\zeta)}{\tan \left(\zeta_{L}\right)}\right) \epsilon(0)+\frac{\sin (\zeta)}{\sin \left(\zeta_{L}\right)} \epsilon(L),
$$

where $\zeta=\frac{1}{2} \kappa X_{1}, \zeta_{L}=\frac{1}{2} \kappa L, \kappa=\sqrt{\kappa^{T} \kappa}$. The constant curvature vector can be extracted from the end values through the formula

$$
\boldsymbol{\kappa}=\frac{1}{\beta_{2} L}\left[\left(\widetilde{\epsilon(L)}-2 \epsilon_{0}(L) \mathbf{I}\right) \epsilon(0)-\left(\widetilde{\epsilon(0)}-2 \epsilon_{0}(0) \mathbf{I}\right) \epsilon(0)\right],
$$

This interpolation is then transformed to the variations in terms of the rotational vector. Details are provided in Crivelli's thesis ${ }^{26}$. 


\section{NUMERICAL EXAMPLES}

The proposed formulation is applied to a set of six problems

\section{Small Deflection Analysis of a Cantilever Beam}

This example evaluates the behavior of our formulation for small rotations and displacements, in which case it should recover the full linear response. A two-dimensional cantilever beam has length $L=5$, cross-section area $A=4.8 \times 10^{-3}$ and moment of inertial $I=4.45 \times 10^{-5}$, with elastic modulus $E=2.1 \times 10^{11}$ and shear modulus $G=8.0775 \times 10^{10}$. The beam is loaded on its free end with a vertical force $P=600$. Two sets of results are reported. The first with the formulation as is, whereas in the second we introduce the residual bending flexibility correction of $\mathrm{McNeal}^{27}$. The purpose of this correction is to accelerate the convergence of the linear $C^{0}$ bending elements, so that linear interpolation functions approximate the behavior of Hermitian cubics. This behavior is desirable since this thin beam can be accurately represented by a Bernoulli beam, for which the analytical solution is indeed cubic.

The numeric results are shown in Table 3 for meshes with increasing number of elements. The second and third columns correspond to the tip deflection and rotation when no correction is introduced while the fourth and fifth columns are the values obtained when the residual bending flexibility is taken into account.

$\begin{array}{ccccc}\begin{array}{c}\text { Number of } \\ \text { Elements }\end{array} & \begin{array}{c}\text { Tip Deflection } \\ \text { w.o. correction }\end{array} & \begin{array}{c}\text { Tip Rotation } \\ \text { w.o. correction }\end{array} & \begin{array}{c}\text { Tip Deflection } \\ \text { w. correction }\end{array} & \begin{array}{c}\text { Tip Rotation } \\ \text { w. correction }\end{array} \\ & & & & \\ 1 & -2.016 \times 10^{-3} & -8.026 \times 10^{-4} & -2.684 \times 10^{-3} & -8.026 \times 10^{-4} \\ 2 & -2.517 \times 10^{-3} & -8.026 \times 10^{-4} & -2.684 \times 10^{-3} & -8.026 \times 10^{-4} \\ 4 & -2.643 \times 10^{-3} & -8.026 \times 10^{-4} & -2.684 \times 10^{-3} & -8.026 \times 10^{-4} \\ 8 & -2.74 \times 10^{-3} & -8.026 \times 10^{-4} & -2.684 \times 10^{-3} & -8.026 \times 10^{-4} \\ 16 & -2.682 \times 10^{-3} & -8.026 \times 10^{-4} & -2.684 \times 10^{-3} & -8.026 \times 10^{-4}\end{array}$

Table 3: Comparison of results for the cantilever beam for the small displacement case.

As expected for a Timoshenko beam element, ${ }^{28}$ the displacements in the first case are too stiff when the mesh is coarse, whereas the tip rotation is accurate. When the residual bending flexibility correction is used, we obtain the exact solution for any mesh. 


\section{Large Displacement and Rotation Analysis}

The second example is one of the few nonlinear beam problem with a simple analytic solution. It consists of a straight cantilever beam loaded with a moment $M$ at its free end, as shown in Figure 2.

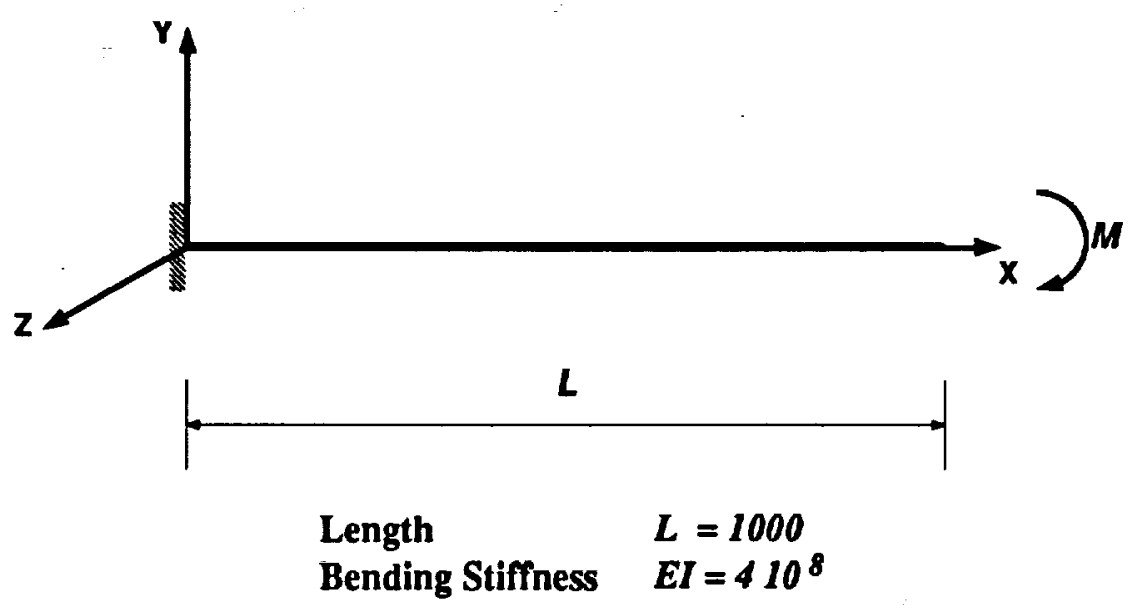

Figure 2: Straight Cantilever Beam Loaded by a Tip Moment. Problem Definition.

The exact solution is a circle of radius $r=E I / M$. The beam has length $L=1000$ and bending stiffness $E I=4 \times 10^{8}$ while the applied moment is $M=2 \pi \times 10^{5}$. The mesh consists of 10 equally-spaced elements and the full load is applied in four increments. Using full Newton, convergence is attained in an average number of four iterations per increment. The deflected shapes for half-load and full-load levels are shown in Figure 3.

\section{Cantilever Beam with Two Transversal Loads}

This example consists of a two-dimensional cantilever beam with two vertical loads, one applied at the free end and the other one close to mid-span, as shown in Figure 4. This problem has been considered by several authors and an analytic solution is available, see e.g. Ebner and Ucciferro. ${ }^{29}$

The cross-section area of this beam is $A=0.2$, while the bending flexibility is $E I=5 \times 10^{6}$ and the shear modulus is $G=1.153846 \times 10^{7}$. The full load is applied in ten equal increments. The structure has been discretized by eight equally spaced finite elements. 


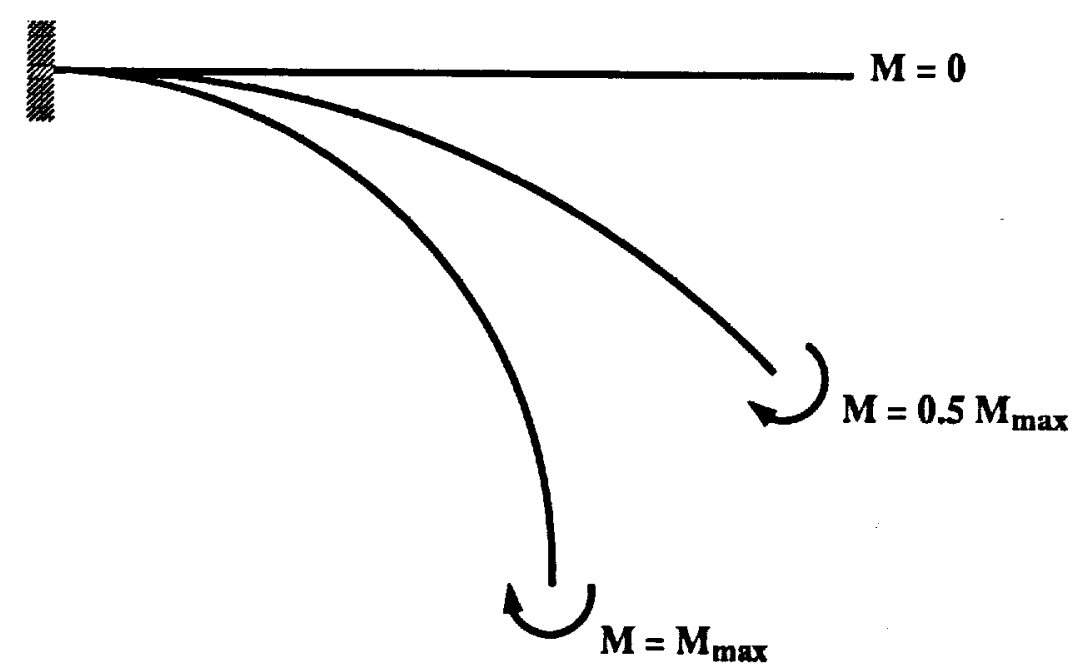

Figure 3: Straight Cantilever Beam Loaded by a Tip Moment. Deformed Shapes.

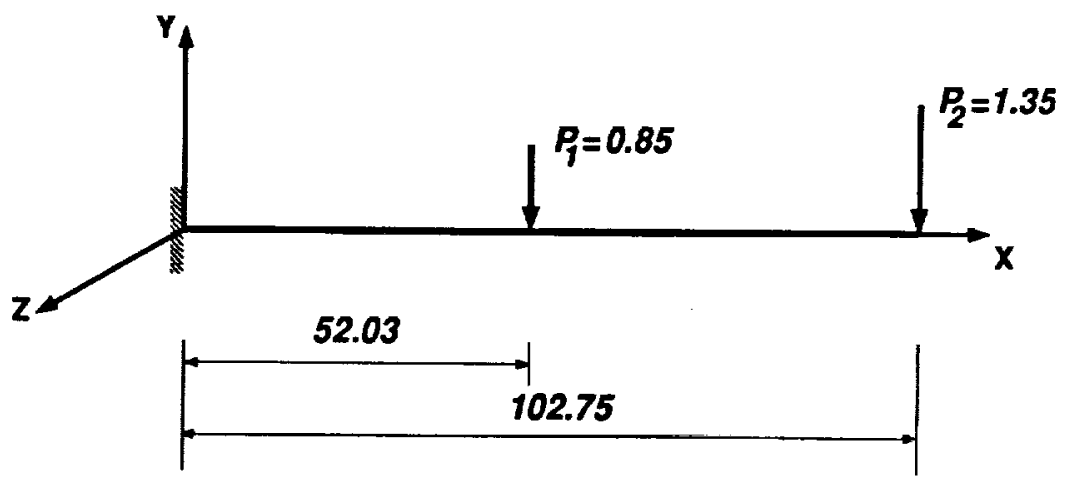

$\begin{array}{lll}\text { Bending Stiffness } & E I=5 \quad 10^{6} \\ \text { Shear Modulus } & G=1.15 \quad 10^{7} \\ \text { Area } & A=0.2\end{array}$

Figure 4: Straight Cantilever Beam Loaded by Two Transversal Loads. Problem Definition.

Table 4 gives the tip displacements and rotation for different meshes and different formulations. The results obtained by the present model are compared to those obtained by Cardona ${ }^{24}$ and to those provided by the analytic solution. Deformed configurations for selected load levels are shown in Figure 5.

Large Displacement 3-D Analysis of 45-Degree Bend 
Formulation

\begin{tabular}{llccc} 
Formulation & & \multicolumn{3}{c}{ Elements } \\
& & 2 & 4 & 8 \\
Cardona & Longitudinal & -28.99 & -30.26 & -30.62 \\
& Transversal & -65.86 & -66.63 & -66.87 \\
& Rotation & -1.1 & -1.06 & -1.05 \\
Present & Longitudinal & -28.99 & -30.26 & -30.62 \\
& Transversal & -65.86 & -66.63 & -66.87 \\
& Rotation & -1.1 & -1.06 & -1.05 \\
Analytic & Longitudinal & -30.75 & -30.75 & -30.75 \\
& Transversal & -66.96 & -66.96 & -66.96 \\
& Rotation & - & - & -
\end{tabular}

Table 4: Comparison of results for the cantilever beam with two transversal loads.

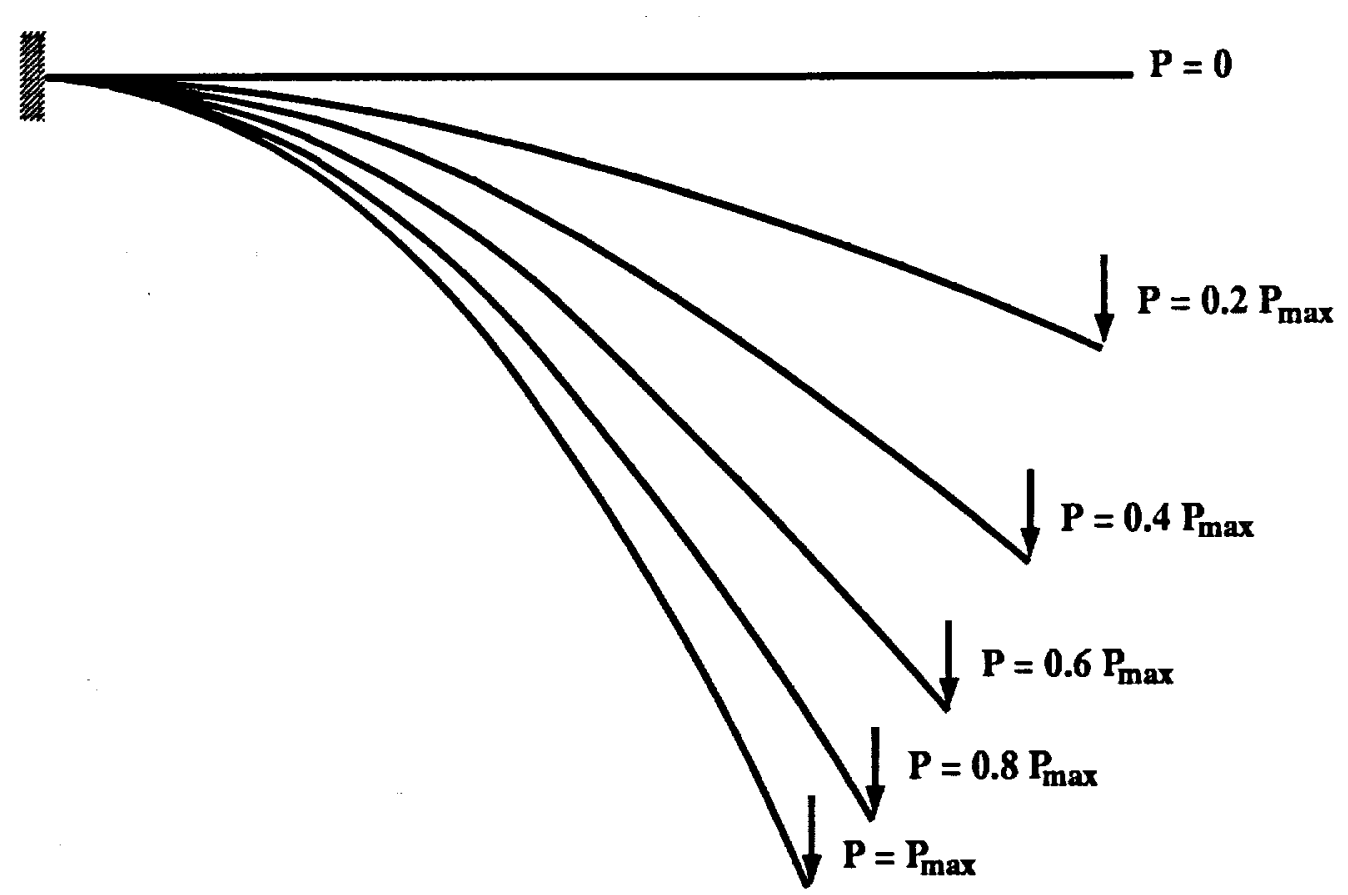

Figure 5: Straight Cantilever Beam Loaded by Two Transversal Loads. Deformed Shapes.

This example has been studied by Bathe and Bolourchi. ${ }^{30}$ It consists of a cantilever 45-degree 
bend lying on a horizontal plane, subjected to an end-load normal to that plane, as shown in Figure 6.

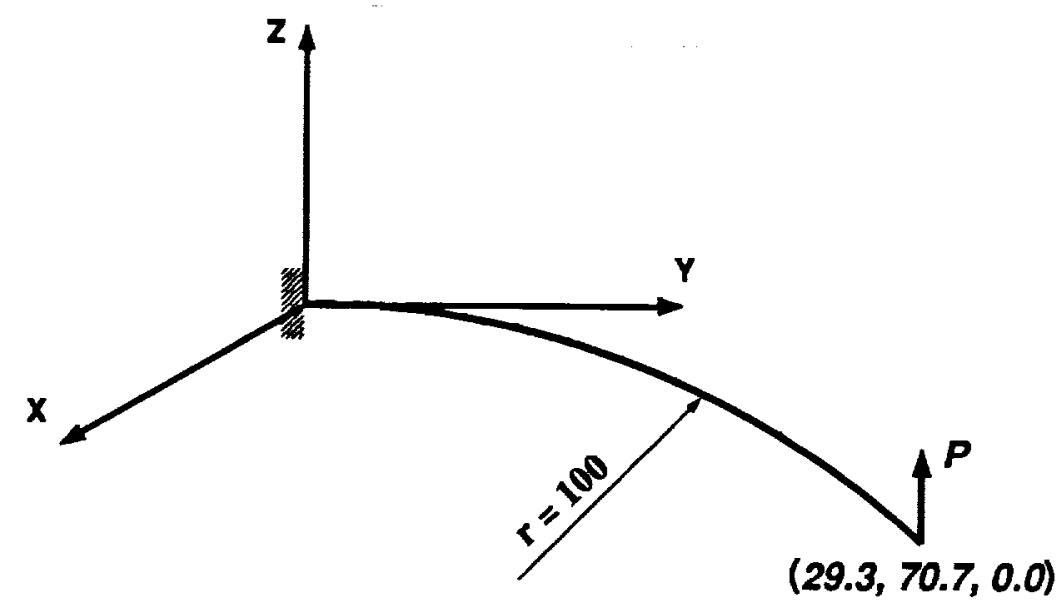

Figure 6: Curved Cantilever Bend Loaded by Tip Load. Problem Definition.

The bend is an arc of a circle of radius $r=100$ and the beam cross-section is a square with sides of unit length. The material has elastic modulus $E=10^{7}$ and a zero Poisson ratio. The finite element mesh consist of 8 straight beam elements. The full load $P=600$ is applied in 6 equal increments.

Table 5 compares the results obtained by different formulations while the deflected shapes corresponding to selected load levels are presented in Figure 7.

\begin{tabular}{lccc} 
Formulation & \multicolumn{3}{c}{ Load } \\
& 300 & 450 & 600 \\
Bathe et.al & $22.33,58.84,40.08$ & $18.62,53.32,48.39$ & $15.79,47.23,53.37$ \\
Simo et.al & $22.50,59.20,39.50$ & & $15.90,47.20,53.40$ \\
Cardona & $22.14,58.64,40.35$ & $18.38,52.11,48.59$ & $15.55,47.04,53.50$ \\
Present & $22.31,58.85,40.08$ & $18.59,53.34,48.39$ & $15.75,47.25,53.37$
\end{tabular}

Table 5: Comparison of results for the 45-degree bend cantilever beam with a transversal tip load. 


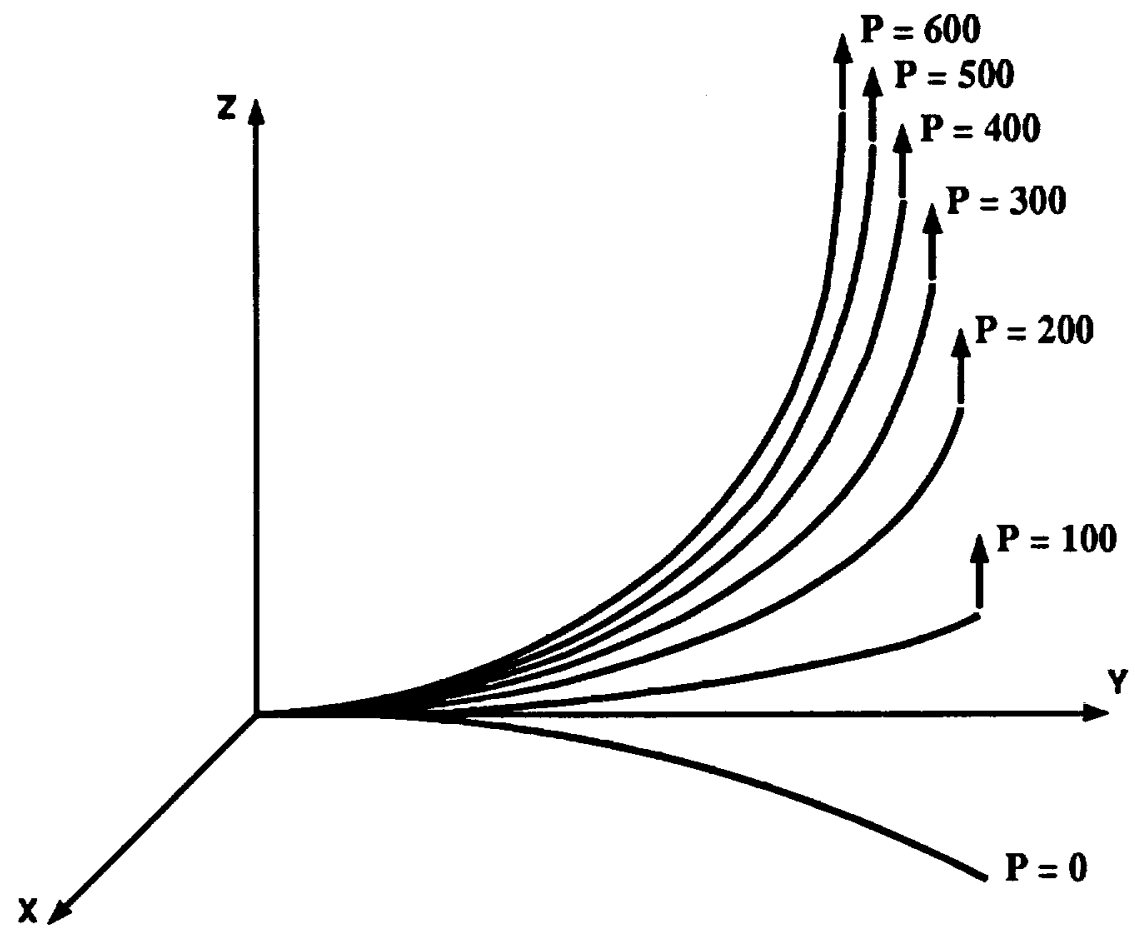

Figure 7: Curved Cantilever Bend Loaded by Tip Load. Deflected Shapes.

This table shows that the result obtained by Cardona ${ }^{24}$ are stiffer whereas those obtained by Simo and $\mathrm{Vu}-\mathrm{Quoc}^{23}$ are more flexible than those obtained by Bathe et.al. ${ }^{30}$ However, these latter results can be taken as reference since they have also been obtained using a more refined mesh. It can be seen that the results of the present formulation agree well with the reference solution.

\section{Williams' Toggle Beam}

This problem consists of two thin plane beams rigidly jointed together and clamped at both ends. The load $P$ is applied at the apex of the structure. The geometry and physical properties are given in Figure 8. Williams ${ }^{31}$ solved this problem analytically by taking into account the effects of finite changes in geometry as well as the effects of the axial force in the flexural stiffness and the flexural modification of the axial stiffness. He also compared his results to experimental data.

This problem was first solved numerically by Wood and Zienkiewicz ${ }^{32}$ using five Total- 


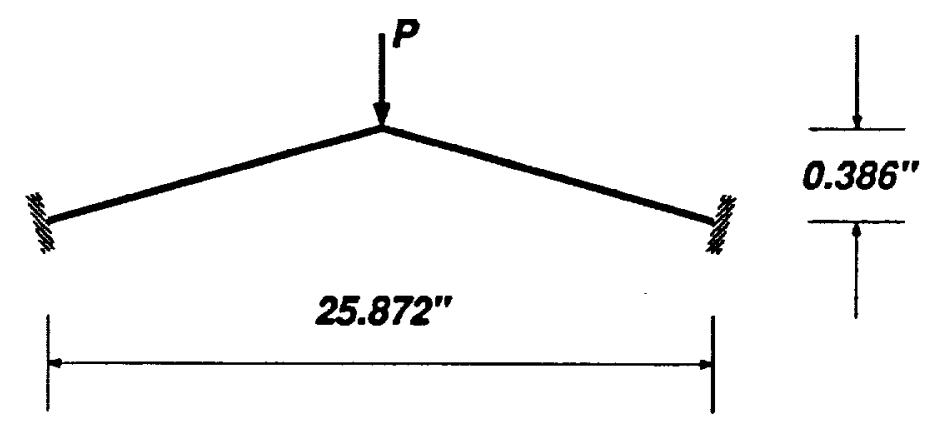

$\begin{array}{lll}\text { Stretching Stiffness } & E A=1.855 \quad 10^{6} \mathrm{lb} \\ \text { Bending Stiffness } & E I=9.27 \quad 10^{3} \mathrm{lb}^{2} \mathrm{in}^{2}\end{array}$

Figure 8: William's Toggle. Problem Definition.

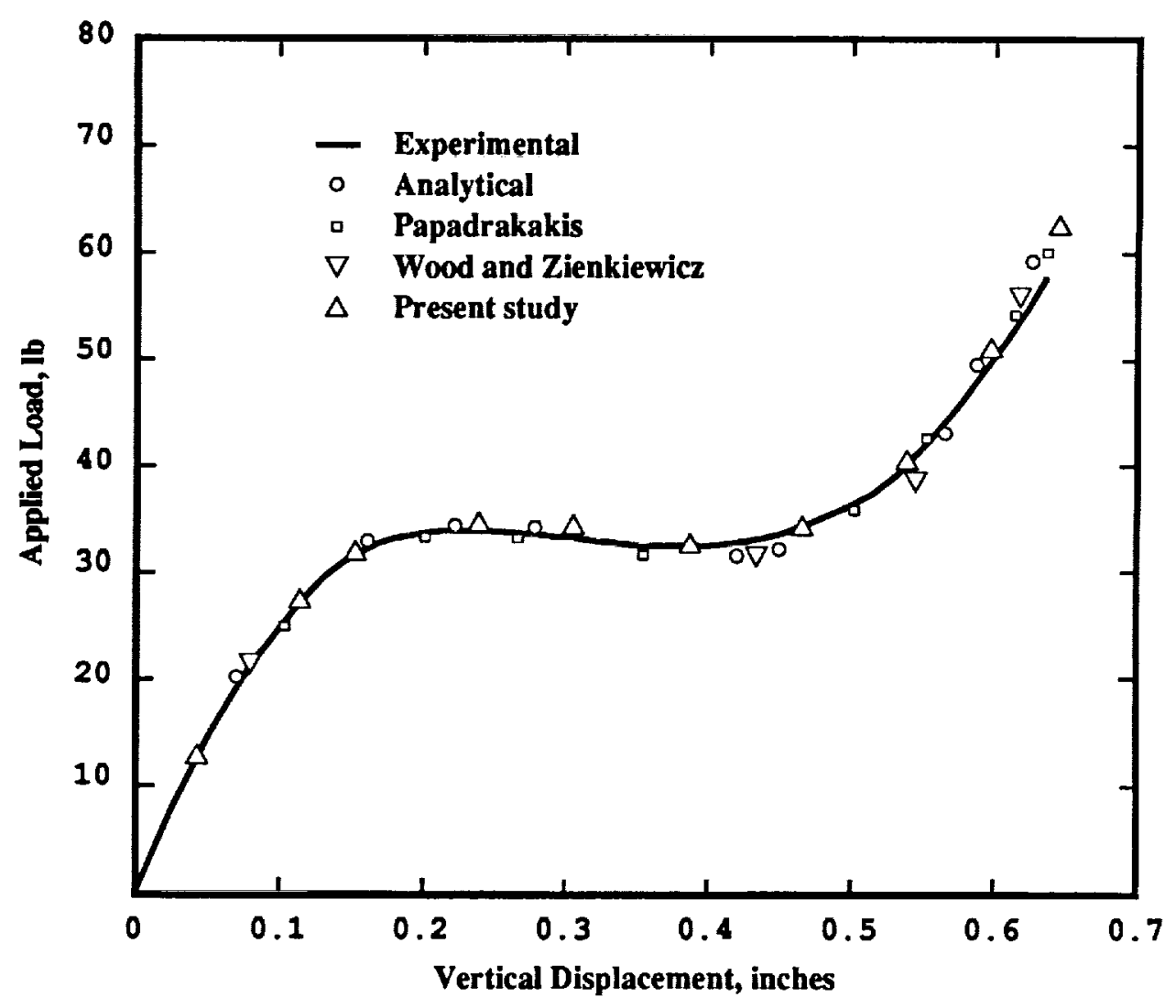

Figure 9: William's Toggle. Load-Deflection curves.

Lagrangian paralinear isoparametric elements per member. In addition, these authors used a 
displacement control strategy to traverse the limit point. This problem was also analyzed by Papadrakakis ${ }^{33}$ using two Euler-Bernoulli beams, formulated under the assumption of large displacements but moderate rotations. Nee and Haldar ${ }^{35}$ solved this problem by using an assumed stress formulation. We have solved this problem using five elements per member together with the residual bending flexibility concept ${ }^{27}$ to approximate the thin beam behavior. Comparisons of the tip deflections versus load obtained by these different formulations are given in Figure 9. We observe a good agreement between our results and experimental measurements.

\section{Twelve Member Frame}

This problem consists of a three-dimensional frame made of twelve members, with half of them laid out as an hexagon, and the other half making up the diagonals of the hexagon. The load is applied on the central node. The geometry and physical properties of the frame are described in Figure 10.
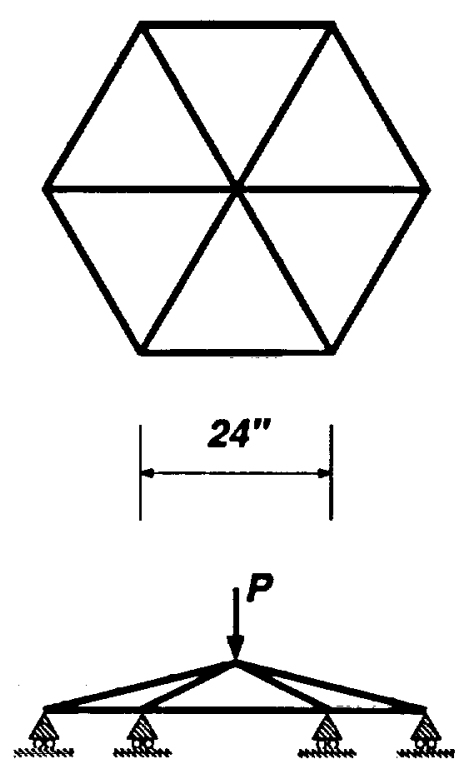

$1.75^{\prime \prime}$

Polar Moment $\quad I_{x}=0.0331 \mathrm{in}^{4}$

Second Moments $\quad I_{y}=I_{z}=0.0203$ in $^{4}$

Young's Modulus $E=439.8 \mathrm{ksi}$

Shear Modulus $\quad G=159 \quad k s i$

Area

$A=0.494 \mathrm{in}^{2}$

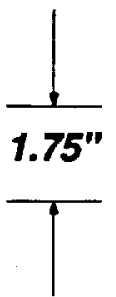

Figure 10: Twelve-Member Frame. Problem Definition.

This frame is simply supported and the supports are allowed to move on the plane normal to the load. To remove the translational rigid body modes and make the problem determinate, 
the central node is restricted against lateral displacement. The frame has been discretized using one finite element for each member of the base and two elements for each diagonal member.

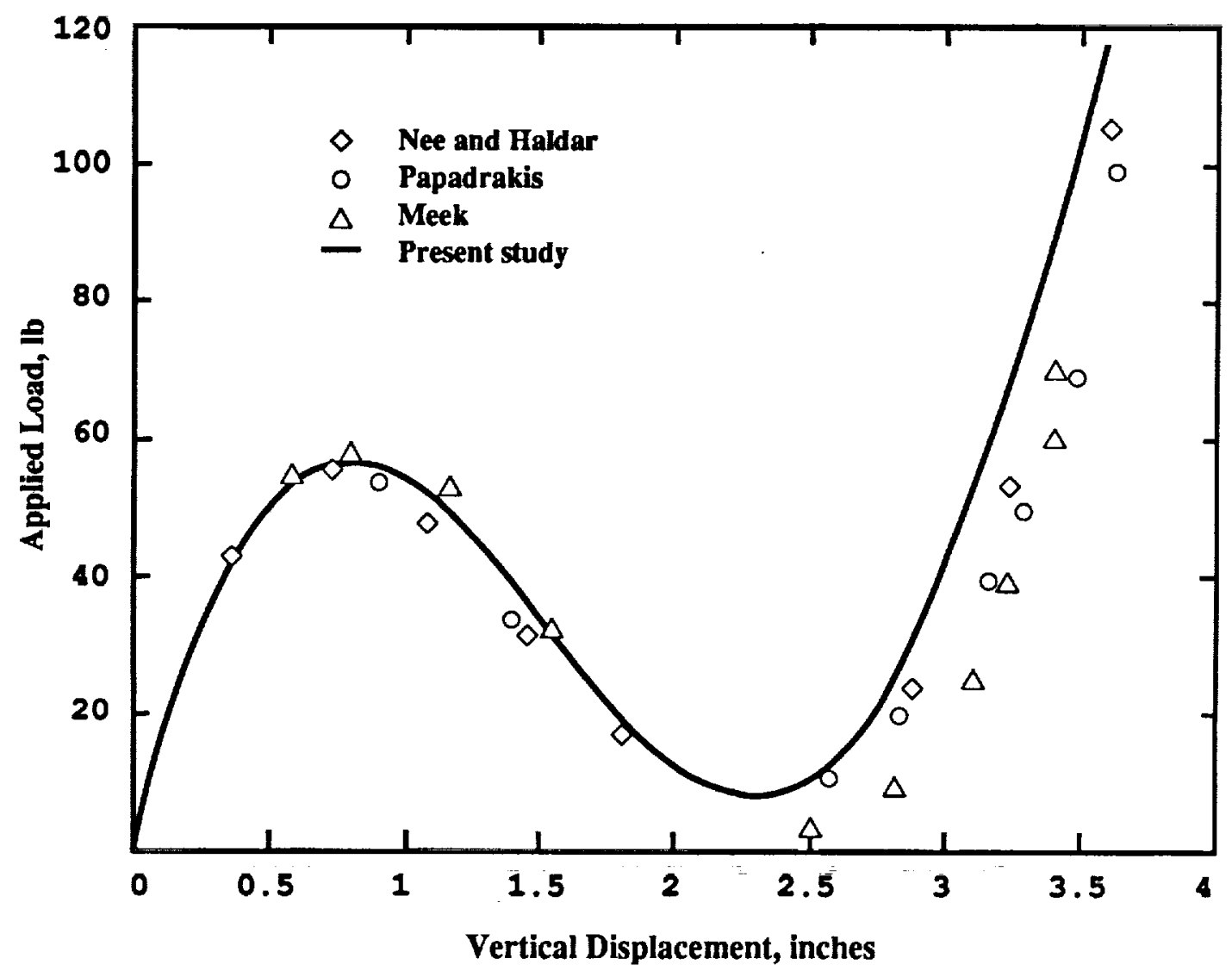

Figure 11: Twelve-Member Frame. Load-Deflection Curves.

The evolution of the deflection of the apex while the load is varied is given in Figure 11. An incremental strategy with step control and a hyperelliptic constraint have been used to traverse the limit point. The results obtained by the present formulation are compared to those obtained by Papadrakakis, ${ }^{33}$ Meek and Tan $^{34}$ and Nee and Haldar. ${ }^{35}$ It can be noticed that the present formulation displays a slightly stiffer behavior, which can be attributed to the presence of the shear stress. The extra stiffness should disappear with more refined meshes.

\section{CONCLUSIONS}

We have constructed and tested a three-dimensional Timoshenko beam element based on the Total Lagrangian description. The element has 12 degrees of freedom: 6 translations and 
6 finite rotations measured by the rotational vector. This particular set of nodal freedoms reduces to the usual choice in small-deflection analysis. This uniformity of treatment allows implementation in standard finite element codes without the necessity of making special provisions to update the rotations. Furthermore, the formulation leads to a symmetric tangent stiffness matrix for arbitrary motions. This attribute is particularly valuable in stability analysis of complex structures, as it allows the use of symmetric eigensolvers near bifurcation points.

The kinematics of the deformation is described using an inertial frame attached to the undeformed configuration of the beam. This kinematic description has a potential advantage in nonlinear dynamic calculations in that the mass matrix remains fixed, with the same sparsity as in small-deflection analysis. The use of Green-Lagrange strains, conjugate PK2 stresses and the absence of hazardous a priori kinematic approximations (beyond those of the Timoshenko beam model) effectively filters out arbitrary rigid body motions, and allows the beam element to capture coupling effects between stretching, bending, torsion and transverse shears within the elastic response regime. These abilities augur well for its future use in highly flexible space structures, where the effect of those couplings can be extremely important in stability, dynamics and control.

The discrete equations have been derived using the staged approach of the CoreCongruential Formulation (CCF). In the innermost level, core equations are obtained at the particle level. These physically transparent equations depend only on the form of the internal energy density. A chain of transformations ensues in which the core equations are referred to three sets of kinematic variables, two pertaining to cross sections and the third one to the finite element nodal degrees of freedom. The choice of finite rotation measure is introduced in the second stage. The choice of finite element interpolation and nodal freedoms is introduced in the last stage. This "nesting" offers obvious advantages in fostering programming modularity and maintaning flexibility as regards to decision changes.

We believe that the main contributions of the present work to computational nonlinear mechanics are as follows.

1. The development of a new symmetric formulation for the analysis of the geometrically nonlinear response of three-dimensional beams that undergo arbitrary rotations. The Total Lagrangian description maintains a fixed reference configuration, which is advantageous in many classes of problems. No special treatment of the rotational degrees of freedoms is required thus simplifying the treatment of boundary conditions. The symmetry and 
freedom-choice attributes simplifies the element implementation into stiffness-based finite element codes.

2. The CCF methodology allows a systematic staged development of the Total Lagrangian element equations that maintains physical visibility. The general core equations display microscopic behavior, and are gradually specialized to macroscopic behavior in the transformation phase. Behavioral approximations may be injected in the initial transformation stages, whereas computational decisions as regards rotational parametrization and element discretization may be deferred to the final transformation stages.

Extensive numerical experiments have been performed to validate and test the present formulation and solution methods. These problems cover a wide range of structural behavior, from plane to three-dimensional structures, including snap-through and nonlinear bifurcation. The ability of the present formulation to deal with large three-dimensional rotations and displacements has been demonstrated. In addition, the beam model approaches the linear beam behavior when the displacements and rotations are small. For very thin beams it is well known that Timoshenko beam models may be stiffer than Euler-Bernouilli models. The residual bending flexibility correction may improve this behavior when the displacements are moderate.

Although the solution method is not a focus of this paper, it is noted that good NewtonRaphson convergence rates have been attained for all the tested problems. This behavior validates the consistency of the residual force and tangent stiffness computations.

\section{ACKNOWLEDGEMENTS.}

The first author acknowledges financial support by the Air Force Office of Scientific Research under Grant F49620-87-C-0074 and the National Science Foundation under Grant 87-17773. The second author acknowledges partial support by NASA Langley Research Center under Grant NAS1-756 and the Center for Space Construction (CSC).

\section{G}

\section{REFERENCES}

1. C. A. Felippa and L. A. Crivelli, 'The core congruential formulation of geometrically nonlinear finite elements', in Computational Nonlinear Mechanics-The State of the Art, ed. by P. Wriggers and W. Wagner, Springer-Verlag, Berlin, 1991, pp. 283-302.

2. S. Rajasekaran and D. W. Murray, 'Incremental finite element matrices', J. Str. Div. 
ASCE, 99, 2423-2438 (1973).

3. R. H. Mallet and P. V. Marcal, 'Finite element analysis of nonlinear structures', J. Str. Div. ASCE, 94, 2081-2105 (1968).

4. C. A. Felippa, Discusion of [2], J. Str. Div. ASCE, 100, 2519-2521 (1974).

5. P. B. Grote, J. C. McMunn and R. Gluck, 'Equations of motion of flexible spacecraft', J. of Spacecraft and Rockets, 8, 561-567 (1971).

6. P. W. Likins, 'Finite element appendage equations for hybrid coordinate dynamic analysis', Int. J. Solids Structures, 8, 709-731 (1972).

7. B. Fraeijs de Veubeke, 'The dynamics of flexible bodies', Int. J. Engng. Sci., 14, 895-913 (1976).

8. J. R. Canavin and P. W. Likins, 'Floating reference frames for flexible spacecraft', J. of Spacecraft, 724-732 (1977).

9. T. B. McDonough, 'Formulation of the global equations of motion of a deformable body', AIAA Journal, 14, 656-660 (1976).

10. R. K. Cavin and A. R. Dusto, 'Hamilton's principle: finite-element methods and flexible body dynamics', AIAA Journal, 15, 1684-1690 (1976).

11. O. P. Agrawal and A. A. Shabana, 'Application of deformable-body mean axis to flexible multibody system dynamics', Comp. Meths. Appl. Mech. Engrg., 56, 217-245, (1986).

12. R. A. Laskin, P. W. Likins and R. W. Longman, 'Dynamical equations of a free-free beam subject to large overall motions', J. of the Astronautical Sciences, 31, 507-528 (1983).

13. T. R. Kane and R. R. Ryan, 'Dynamics of a cantilever beam attached to a moving base', Journal of Guidance, Control and Dynamics, 10, 139-151 (1987).

14. L. Meirovitch and R. D. Quinn, 'Equations of motion for maneuvering flexible spacecraft', Journal of Guidance, Control and Dynamics, 10, 453-465 (1987).

15. R. D. Quinn and L. Meirovitch, 'Maneuver and vibration control of SCOLE', Journal of Guidance, Control and Dynamics, 11, 542-553 (1988).

16. K. C. Park, 'Flexible beam dynamics for space structures: Formulation', Center for Space Structures and Controls, University of Colorado, Boulder (1987).

17. J. D. Downer, 'A computational procedure for the dynamics of flexible beams within multibody systems', PhD. Thesis, Department of Aerospace Engineering Sciencies, Uni- 
versity of Colorado, 1990.

18. T. Belytschko and B. J. Hsieh, 'Nonlinear transient finite element analysis with convected coordinates', Int. J. Numer. Meth. Engrg., 7, 255-271 (1973).

19. T. Belytschko, L. Schwer and M.J. Klein, 'Large displacement, transient analysis of space frames', Int. J. Numer. Meth. Engrg., 11, 65-84 (1977).

20. C. C. Rankin, 'Consistent linearization of the element-independent corotational formulation for the structural analysis of general shells', NASA Report 278428, Lockheed Palo Alto Research Laboratory, 1988.

21. B. Nour-Omid and C. C. Rankin, 'Finite rotation analysis and consistent linearization using projectors', Comp. Meths. Appl. Mech. Engrg., 93, 353-384 (1991).

22. J. C. Simo, 'A finite strain beam formulation. Part I: The three dimensional dynamic problem', Comp. Meths. Appl. Mech. Engrg., 49, 55-70 (1985).

23. J. C. Simo and L. Vu-Quoc, 'A three-dimensional finite strain rod model. Part II: Computational aspects', Comp. Meths. Appl. Mech. Engrg., 58, 79-116 (1986).

24. A. Cardona, 'An integrated approach to mechanism analysis', Doctoral Thesis, Universite de Liege, 1989.

25. J. D. Downer, K. C. Park and J. C. Chiou, 'A computational procedure for multibody systems including flexible beam dynamics', Proc. AIAA Dynamics Specialists Conference, Long Beach, April 4-6, 1990, also Comp. Meths. Appl. Mech. Engrg., in press..

26. L. A. Crivelli, 'A total-lagrangian beam element for analysis of nonlinear space structures', PhD. Thesis, Department of Aerospace Engineering Sciencies, University of Colorado, 1991.

27. R. H. MacNeal, 'A simple quadrilateral shell element', Computer \& Structures, 8, 175183 (1978).

28. T. J. R. Hughes, The Finite Element Method, Prentice Hall, N.J., 1987.

29. A. M. Ebner and J. J. Ucciferro, 'A theoretical and numerical comparison of elastic nonlinear finite element methods', Computer \& Structures, 2, 1043-1061 (1972).

30. K. J. Bathe and S. Bolourchi, 'Large displacement analysis of three-dimensional beam structures', Int. J. Numer. Meth. Engrg., 14 961-986 (1979).

31. F. W. Williams, 'An approach to the nonlinear behaviour of the members of a rigid jointed plane framework with finite deflection', Quart. J. Mech. Appl. Math., 17, 451469 (1964). 
32. R. D. Wood and O. C. Zienkiewicz, 'Geometrically nonlinear finite element analysis of beams, frames, arches and axisymmetric shells', Computer \& Structures, 7, 725-735 (1977).

33. M. Papadrakakis, 'Post-buckling analysis of spatial structures by vector iteration methods', Computer \& Structures, 14, 393-402 (1981).

34. J. L. Meek and H. S. Tan, 'Geometrically nonlinear analysis of space frames by an incremental iterative technique', Comp. Meths. Appl. Mech. Engrg., 47, 261-282 (1984).

35. K. M. Nee and A. Haldar, 'Elastoplastic nonlinear post-buckling analysis of partially restrained space structures', Comp. Meths. Appl. Mech. Engrg., 71, 69-97 (1988). 


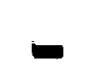

$=$

$\bar{\equiv}$

$\square$

$\overline{\bar{E}}$

$\overline{\overline{\underline{a}}}$

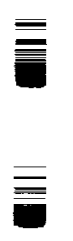

$\overline{=}$

$\overline{\bar{\sigma}}$

$=$

-

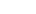

$=$

$\underline{\underline{\underline{\underline{\underline{\underline{ }}}}}}$

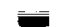

$\overline{\bar{D}}$

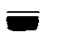

$\bar{\Xi}$

 